\title{
Quantum cohomology of toric blowups and Landau-Ginzburg correspondences
}

\author{
Pedro Acosta and Mark Shoemaker
}

\begin{abstract}
We establish a genus zero correspondence between the equivariant Gromov-Witten theory of the Deligne-Mumford stack $\left[\mathbb{C}^{N} / G\right]$ and its blowup at the origin. The relationship generalizes the crepant transformation conjecture of Coates-Iritani-Tseng and Coates-Ruan to the discrepant (non-crepant) setting using asymptotic expansion. Using this result together with quantum Serre duality and the multiple log-canonical correspondence, we prove genus zero correspondences between Landau-Ginzburg theory and the Gromov-Witten theory of non-Calabi-Yau hypersurfaces.
\end{abstract}

\section{Introduction}

Given a birational map $f: \mathcal{Y} \rightarrow \mathcal{X}$ between smooth complex varieties (or orbifolds), it is natural to ask if there exists a relationship between the Gromov-Witten theories of $\mathcal{X}$ and $\mathcal{Y}$. This question has a long history and has been studied by many authors (see, for instance, [Gat01, Hu00, BG09, CIT09, CR13, HH15, Rua99]). For example, Hu proved in the symplectic setting that if $f$ is the blowup of a smooth point or a curve, many of the Gromov-Witten invariants remain unchanged.

In general, however, the Gromov-Witten theories of $\mathcal{X}$ and $\mathcal{Y}$ will not be equal, and so the question remains whether or not it is possible to relate the two theories in any systematic manner. In this paper, we propose such a correspondence in terms of asymptotic expansion and provide a proof in the case where $f: \mathcal{Y} \rightarrow \mathcal{X}$ is a blowup of $\left[\mathbb{C}^{N} / G\right]$ at the origin.

\subsection{Asymptotic expansion}

1.1.1 The crepant case. A key insight comes from mirror symmetry, which suggests that for certain $K$-equivalent varieties, the relationship between their Gromov-Witten theories is given by analytic continuation and quantization. To be more precise, generating functions of GromovWitten invariants of $\mathcal{X}$ should be related to generating functions of Gromov-Witten invariants of $\mathcal{Y}$ by analytic continuation and a (possibly quantized) symplectic transformation. This relationship has been dubbed the crepant resolution (or, more generally, the crepant transformation) conjecture and may be viewed as an instance of the McKay correspondence (see [Rei02]) for GromovWitten theory. The conjecture was proposed in various levels of generality by Li-Ruan [LR01],

Received 8 April 2017, accepted in final form 3 May 2017.

2010 Mathematics Subject Classification 14N35, $14 \mathrm{E} 05$.

Keywords: crepant transformation conjecture, FJRW theory, LG/CY correspondence.

This journal is (C) Foundation Compositio Mathematica 2018. This article is distributed with Open Access under the terms of the Creative Commons Attribution Non-Commercial License, which permits non-commercial reuse, distribution, and reproduction in any medium, provided that the original work is properly cited. For commercial re-use, please contact the Foundation Compositio Mathematica.

Mark Shoemaker was partially supported by NSF RTG Grant DMS-1246989. 


\section{P. Acosta And M. Shoemaker}

Bryan-Graber [BG09], Coates-Corti-Iritani-Tseng [CIT09], and Coates-Ruan [CR13]. At this point, the conjecture is well studied and has been verified for a wide class of examples. For genus zero correspondences, see for instance [BG09, CIT09, CIJ14, LLQW16, GW12], and for higher genus, see [CI14, BCR13, ILLW12, Zho08].

1.1.2 The general case. An important ingredient in the crepant transformation conjecture is the analytic continuation of generating functions of the respective invariants. Thus a necessary component in this conjecture is that these generating functions, a priori given as power series in certain formal variables, are in fact analytic, at least in some of the variables.

This analyticity, so crucial in the above conjecture, is the first thing to fail for a general birational map $f: \mathcal{Y} \rightarrow \mathcal{X}$. In many cases, one generating function will be analytic with an essential singularity at infinity, while the other generating function has radius of convergence equal to zero. A solution to this obstacle was discovered by the first author in [Aco14] in the context of Landau-Ginzburg correspondences (see Section 1.1.3). It was realized that in this context, analytic continuation should be replaced by power series asymptotic expansion (see Section 5).

Our first main theorem concerns the case where $f: \mathcal{Y} \rightarrow \mathcal{X}$ is a weighted blowup of $\mathcal{X}=$ $\left[\mathbb{C}^{N} / G\right]$ at the origin. The precise assumptions on $\mathcal{X}$ and $f$ are given in Section 4 . In the following, let $|\mathcal{X}|$ denote the coarse space of $\mathcal{X}$, and let $|f|: \mathcal{Y} \rightarrow|\mathcal{X}|$ denote the map induced by $f$. The discrepancy of $|f|$ is defined to be the coefficient of the exceptional divisor in $K_{\mathcal{Y}}-f^{*}\left(K_{|\mathcal{X}|}\right)$.

Theorem 1.1 (see Theorems 5.1 and 5.10). When $|f|: \mathcal{Y} \rightarrow|\mathcal{X}|$ has positive discrepancy, the quantum cohomology of $\mathcal{Y}$ fully determines the quantum cohomology of $\mathcal{X}$. When $|f|: \mathcal{Y} \rightarrow|\mathcal{X}|$ has negative discrepancy, the quantum cohomology of $\mathcal{X}$ fully determines the quantum cohomo$\log y$ of $\mathcal{Y}$.

The goal of this paper is to introduce the tool of asymptotic expansion as a way of relating generating functions of Gromov-Witten invariants of birational spaces. We restrict ourselves here to the specific case described above for two reasons. First, it allows us to state and prove the correspondence in a simple situation with (relatively) light notation. Second, it is exactly the setting of relevance for Landau-Ginzburg correspondences, which we prove as an application of Theorems 5.1 and 5.10 and explain below.

In a sequel [AS16], we prove a general version of the above theorem in the context of toric wall-crossing.

1.1.3 Landau-Ginzburg correspondences. Given a non-degenerate quasi-homogeneous polynomial $W=W\left(X_{1}, \ldots, X_{N}\right)$ and an admissible group $G$ (see [FJR13]), one can define the corresponding FJRW invariants, which may be viewed as the analog of Gromov-Witten invariants for the singular space $\{W=0\} \subset\left[\mathbb{C}^{N} / G\right]$. They are given as integrals over a cover of the moduli space of stable curves and obey many of the same axioms as Gromov-Witten invariants.

One may also view the pair $(W, G)$ as defining a hypersurface $\mathcal{Z}:=\{W=0\} \subset \mathbb{P}(G)$, where $\mathbb{P}(G)$ denotes a suitable quotient of weighted projective space. Again inspired by mirror symmetry, the Landau-Ginzburg/Calabi-Yau correspondence is a conjectural relationship between the FJRW invariants of the pair $(W, G)$ and the Gromov-Witten theory of $\mathcal{Z}$ in the case where $\mathcal{Z}$ is a Calabi-Yau variety. It was first verified for the quintic hypersurface in $\mathbb{P}^{4}$ in genus zero [CR10] and has since been extended to all cases where $W$ is a Fermat polynomial and $G \leqslant \mathrm{SL}_{N}(\mathbb{C})$ [LPS16]. 


\section{QUANTUM COHOMOLOGY OF BLOWUPS}

In [Aco14], the first author extended this correspondence to the case where $\mathcal{Z}$ was either a Fano or general type hypersurface in weighted projective space. Our second main theorem generalizes this to the case where $W$ is a Fermat polynomial:

$$
W=X_{1}^{d / c_{1}}+\cdots+X_{N}^{d / c_{N}}
$$

with $\operatorname{gcd}\left(c_{1}, \ldots, c_{N}\right)=1$ and $W$ gives a section of a line bundle $\mathcal{L}$ pulled back from the coarse space of $\mathbb{P}(G)$. The condition $\sum_{j=1}^{N} c_{j}=d$ is exactly the condition necessary to guarantee that $\mathcal{Z}$ is Calabi-Yau. Here, we deal with the other cases.

Theorem 1.2 (see Theorems 6.7 and 6.10). If $\sum_{j=1}^{N} c_{j}-d>0$, the quantum cohomology of the hypersurface $\mathcal{Z}$ determines the quantum cohomology of the FJRW theory of $(W, G)$. If $\sum_{j=1}^{N} c_{j}-d<0$, the quantum cohomology of the FJRW theory of $(W, G)$ determines the quantum cohomology of $\mathcal{Z}$.

\subsection{What is the correspondence?}

In the statements of the above theorems, we have been intentionally vague in saying how the quantum cohomology of one theory determines the other. The precise statement is phrased in terms of generating functions of invariants. Consider the case where $f: \mathcal{Y} \rightarrow-\mathcal{X}$ is the blowup from above. We consider certain generating functions, $I^{\mathcal{Y}}(q, z)$ and $I^{\mathcal{X}}(t, z)$, of genus zero GromovWitten invariants of $\mathcal{X}$ and $\mathcal{Y}$ (see Section 3.3). It is known that these functions fully determine the genus zero Gromov-Witten theory of $\mathcal{X}$ and $\mathcal{Y}$, respectively. In the case where $\mathcal{X}$ and $\mathcal{Y}$ are $K$-equivalent, the crepant transformation conjecture is proven by showing that under the identification $t=q^{-d}$, there exists a unique linear transformation $L$ such that the analytic continuation of $L \cdot I^{\mathcal{Y}}(q, z)$ to a neighborhood of $t=0$ yields the function $I^{\mathcal{X}}(t, z)$.

Now, consider the case where $|f|: \mathcal{Y} \rightarrow|\mathcal{X}|$ has positive discrepancy. Then $I^{\mathcal{X}}(t, z)$ will no longer be an analytic function. The correct analog of Theorem 1.2 is to show that there exists a unique linear transformation $L$ such that the power series asymptotic expansion of $L \cdot I^{\mathcal{Y}}(q, z)$ recovers $I^{\mathcal{X}}(t, z)$. Because the power series asymptotic expansion of a function is uniquely determined (if it exists), this implies that $I^{\mathcal{X}}(t, z)$ is uniquely determined by $I^{\mathcal{Y}}(q, z)$. Because the $I$-functions fully determine the respective genus zero Gromov-Witten theories, this shows that the Gromov-Witten theory of $\mathcal{Y}$ fully determines the Gromov-Witten theory of $\mathcal{X}$. It is within this framework that all of our theorems are proven.

In a similar spirit, Iritani has also announced results relating the genus zero Gromov-Witten theory of $f: \mathcal{Y} \rightarrow \mathcal{X}$ when $f$ is a blowup of a toric variety. His results, however, are phrased in terms of the quantum connection and so are not directly related to our statements on generating functions. It will be interesting to understand the relationship between these respective frameworks.

\section{An example}

Consider the space $\left[\mathbb{C}^{3} / \mathbb{Z}_{2}\right]$ with the diagonal action and its resolution $\operatorname{Tot}\left(\mathscr{O}_{\mathbb{P}^{2}}(-2)\right)$. In this section, we will give an explicit computation of the relationship between the genus zero GromovWitten theories of these spaces. This will serve to illustrate the general principle in a simple example. The $I$-function for the line bundle $\operatorname{Tot}\left(\mathscr{O}_{\mathbb{P}^{2}}(-2)\right)$ is given by

$$
I^{\operatorname{Tot}\left(\mathscr{O}_{\mathbb{P}^{2}}(-2)\right)}(q, z):=z \sum_{n \geqslant 0} q^{n+H / z} \frac{\prod_{l=0}^{2 n-1}(-2(H+\lambda)-l z)}{\prod_{l=1}^{n}(H+l z)^{3}},
$$




\section{P. Acosta And M. Shoemaker}

where $H$ satisfies $H^{3}=0$ and $\lambda$ is the equivariant parameter of the torus action of $\mathbb{C}^{*}$ on $\operatorname{Tot}\left(\mathscr{O}_{\mathbb{P}^{2}}(-2)\right)$. A simple ratio test argument shows that up to a choice of branch cut for $\log (q)$, this series is holomorphic everywhere in the complex plane. From this it follows that the $I$ function cannot be extended holomorphically to the point $q=\infty$. It is still possible, however, to try to understand its asymptotic behavior as $q \rightarrow \infty$. The main claim of this example is that up to a linear transformation, the asymptotic behavior of $I^{\operatorname{Tot}\left(\mathscr{O}_{\mathbb{P}}(-2)\right)}$ as $q \rightarrow \infty$ is given by the $I$-function of $\left[\mathbb{C}^{3} / \mathbb{Z}_{2}\right]$, that is, there exists a unique linear transformation $L: H_{\mathbb{C}^{*}}^{*}\left(\mathscr{O}_{\mathbb{P}^{2}}(-2)\right) \rightarrow$ $H_{\mathrm{CR}, \mathbb{C}^{*}}^{*}\left(\left[\mathbb{C}^{3} / \mathbb{Z}_{2}\right]\right)$ such that

$$
L \cdot I^{\operatorname{Tot}\left(\mathscr{O}_{\mathbb{P}^{2}}(-2)\right)}(q, z=1) \sim I^{\left[\mathbb{C}^{3} / \mathbb{Z}_{2}\right]}\left(t=q^{-1 / 2}, z=1\right) \quad \text { as } q \rightarrow \infty .
$$

Here $I^{\left[\mathbb{C}^{3} / \mathbb{Z}_{2}\right]}$ is the $I$-function for the quotient $\left[\mathbb{C}^{3} / \mathbb{Z}_{2}\right]$ given by the formal series

$$
I^{\left[\mathbb{C}^{3} / \mathbb{Z}_{2}\right]}(t, z):=z \sum_{k=0,1} \sum_{m \geqslant 0} t^{2 m+k+2 \lambda / z} \frac{\prod_{l=0}^{m-1}(-\lambda-(k / 2+l) z)^{3}}{z^{2 m+k}(2 m+k) !} \mathbb{1}_{k},
$$

where $\mathbb{1}_{0}$ and $\mathbb{1}_{1}$ are the fundamental classes of the untwisted and twisted sectors of the inertia stack of $\left[\mathbb{C}^{3} / \mathbb{Z}_{2}\right]$, respectively. The lesson we rescue from this result is that even though we no longer have an equivalence of local theories as in the crepant case, it is possible to obtain the genus zero theory of the singular quotient $\left[\mathbb{C}^{3} / \mathbb{Z}_{2}\right]$ in terms of the asymptotics of the genus zero theory of its blowup $\operatorname{Tot}\left(\mathscr{O}_{\mathbb{P}^{2}}(-2)\right)$.

To establish equation (2.1), we employ a technique known as Borel summation (for a simple example, see [Mil06, $\S 6.3 .3$, pp. 246-249]), which consists of the following steps:

Step 1: Regularize the I-function for the quotient stack $\left[\mathbb{C}^{3} / \mathbb{Z}_{2}\right]$. We define $I_{\mathrm{reg}}(\tau)$ as

$$
I_{\mathrm{reg}}(\tau):=\sum_{k=0,1} \sum_{m \geqslant 0} \tau^{m+k / 2+\lambda} \frac{\prod_{l=0}^{m-1}(-\lambda-(k / 2+l))^{3}}{\Gamma(1+m+k / 2+\lambda)(2 m+k) !} \mathbb{1}_{k} .
$$

This series defines a holomorphic function for $\tau$ in the disk of radius 4 centered at the origin. It is also straightforward to check that $I_{\mathrm{reg}}(\tau)$ satisfies the following linear differential equation:

$$
\left[\tau\left(\tau \frac{\mathrm{d}}{\mathrm{d} \tau}\right)^{3}+\left(2 \tau \frac{\mathrm{d}}{\mathrm{d} \tau}-2 \lambda\right)\left(2 \tau \frac{\mathrm{d}}{\mathrm{d} \tau}-2 \lambda-1\right)\left(\tau \frac{\mathrm{d}}{\mathrm{d} \tau}\right)\right] I_{\text {reg }}(\tau)=0
$$

This differential operator has singular points for $\tau \in\{0,-4, \infty\}$, all of which are regular. It follows that $I_{\text {reg }}(\tau)$ can be analytically continued in the region of the complex plane defined by $|\arg \tau|<\pi$.

Step 2: Apply a Laplace transformation to $I_{\mathrm{reg}}(\tau)$ and use Watson's lemma. Since $I_{\mathrm{reg}}(\tau)$ can be analytically continued to $\tau=\infty$, the following Laplace integral defines a holomorphic function:

$$
\mathbb{I}(q):=q \mathcal{L}\left(I_{\mathrm{reg}}\right)(q):=q \int_{0}^{\infty} e^{-q \tau} I_{\mathrm{reg}}(\tau) \mathrm{d} \tau,
$$

where the ray along which we integrate avoids the singular point $\tau=-4$. As a consequence of Watson's lemma [Mil06], we obtain the following asymptotic expansion for $\mathbb{I}(q)$ :

$$
\begin{aligned}
\mathbb{I}(q) & \sim \sum_{k=0,1} \sum_{m \geqslant 0} q^{-m-k / 2-\lambda} \frac{\prod_{l=0}^{m-1}(-\lambda-(k / 2+l))^{3}}{(2 m+k) !} \mathbb{1}_{k} \\
& =I^{\left[\mathbb{C}^{3} / \mathbb{Z}_{2}\right]}\left(t=q^{-1 / 2}, z=1\right)
\end{aligned}
$$




\section{QUANTUM COHOMOLOGY OF BLOWUPS}

as $q \rightarrow \infty$. The upshot of this step is that we have constructed a holomorphic function $\mathbb{I}(q)$ whose asymptotic expansion is given by the $I$-function of $\left[\mathbb{C}^{3} / \mathbb{Z}_{2}\right]$.

Step 3: Show that $\mathbb{I}(q)$ satisfies the Picard-Fuchs equation of $\operatorname{Tot}\left(\mathscr{O}_{\mathbb{P}^{2}}(-2)\right)$. Expanding equation (2.2), we obtain

$$
\left[\left(\tau^{3}+4 \tau^{2}\right) \frac{\mathrm{d}^{3}}{\mathrm{~d} \tau^{3}}+\left(3 \tau^{2}+(10-8 \lambda) \tau\right) \frac{\mathrm{d}^{2}}{\mathrm{~d} \tau^{2}}+(\tau+(2-2 \lambda)(2-2 \lambda-1)) \frac{\mathrm{d}}{\mathrm{d} \tau}\right] I_{\operatorname{reg}}(\tau)=0 .
$$

Applying a Laplace transform to this yields

$$
-\frac{\mathrm{d}^{3}}{\mathrm{~d} q^{3}}\left(q^{2} \mathbb{I}(q)\right)+\frac{\mathrm{d}^{2}}{\mathrm{~d} q^{2}}\left(3 q \mathbb{I}(q)+4 q^{2} \mathbb{I}(q)\right)-\frac{\mathrm{d}}{\mathrm{d} q}(\mathbb{I}(q)+(10-8 \lambda) q \mathbb{I}(q))+(2-2 \lambda)(1-2 \lambda) \mathbb{I}(q)=0
$$

(where we have assumed $\Re(\lambda) \geqslant 3$ ), which in turn is equivalent to

$$
\left[\left(q \frac{\mathrm{d}}{\mathrm{d} q}\right)^{3}-q\left(-2 q \frac{\mathrm{d}}{\mathrm{d} q}-2 \lambda\right)\left(-2 q \frac{\mathrm{d}}{\mathrm{d} q}-2 \lambda-1\right)\right] \mathbb{I}(q)=0 .
$$

This is precisely the Picard-Fuchs equation satisfied by $I^{\operatorname{Tot}\left(\mathscr{O}_{\mathbb{P}^{2}}(-2)\right)}(q, z=1)$. Since the components of $I^{\operatorname{Tot}\left(\mathscr{O}_{\mathbb{P}} 2(-2)\right)}$ are a complete set of solutions to this differential equation, there exists a unique linear transformation $L$ satisfying $L \cdot I^{\operatorname{Tot}\left(\mathscr{O}_{\mathbb{P}^{2}}(-2)\right)}(q, z=1)=\mathbb{I}(q)$. Equation $(2.1)$ follows from this.

Remark 2.1. In order to compute the asymptotic expansion of $I^{\mathcal{Y}}$, we had to set $z$ equal to 1 in the $I$-functions. We can recover the powers of $z$ in $I^{\mathcal{X}}$ by means of the following procedure. Define a grading operator $\mathbf{G r}$ by $\mathbf{G r}(\lambda)=\lambda, \mathbf{G r}\left(\mathbb{1}_{k}\right)=\frac{3}{2} \mathbb{1}_{k}$; then

$$
I^{\left[\mathbb{C}^{3} / \mathbb{Z}_{2}\right]}(t, z)=z^{1-\mathbf{G r}} z^{-\lambda} I^{\left[\mathbb{C}^{3} / \mathbb{Z}_{2}\right]}\left(t z^{1 / 2}, 1\right) .
$$

Remark 2.2. Equation (2.1) and Birkhoff factorization imply that the genus zero Gromov-Witten invariants of $\left[\mathbb{C}^{3} / \mathbb{Z}_{2}\right]$ are completely determined by the genus zero Gromov-Witten invariants of $\operatorname{Tot}\left(\mathscr{O}_{\mathbb{P}^{2}}(-2)\right)$.

\section{Gromov-Witten theory}

Here, we review the basic definitions of (orbifold) Gromov-Witten theory and set notation. For a reference, see [AGV08] in the algebraic setting or [CR02] in the symplectic.

\subsection{Notation}

Let $\mathcal{X}$ denote a smooth Deligne-Mumford stack with an equivariant action by a torus $T \cong\left(\mathbb{C}^{*}\right)^{r}$. Assume that the fixed-point locus of $\mathcal{X}$ is projective. Let $H_{\mathrm{CR}, T}^{*}(\mathcal{X})=H_{\mathrm{CR}, T}^{*}(\mathcal{X} ; \mathbb{C})$ denote the equivariant Chen-Ruan orbifold cohomology of $\mathcal{X}$ [CR04]. The cohomology $\operatorname{ring} H_{\mathrm{CR}, T}^{*}(\mathcal{X})$ is a module over $R_{T}:=H_{T}^{*}(\mathrm{pt})$. Let $S_{T}$ denote the localization of $R_{T}$ with respect to the set of non-zero homogeneous elements.

Recall that as a vector space, $H_{\mathrm{CR}, T}^{*}(\mathcal{X}) \cong H_{T}^{*}(I \mathcal{X})$, where $I \mathcal{X}$ denotes the inertia stack, parametrizing pairs $(x, g)$ where $x$ is a point in $\mathcal{X}$ and $g \in G_{x}$ is an element of the isotropy group of $x$. The inertia stack $I \mathcal{X}$ is a disjoint union of connected components, $I \mathcal{X}=\coprod_{v \in V} \mathcal{X}_{v}$, where each twisted sector $\mathcal{X}_{v}$ may be identified with a closed substack of $\mathcal{X}$. There is a distinguished component $\mathcal{X}_{\text {id }}$ corresponding to the points $(x, \mathrm{id})$ which is isomorphic to $\mathcal{X}$ itself. We call this 


\section{P. Acosta And M. Shoemaker}

the untwisted sector of $I \mathcal{X}$. Thus, as a vector space,

$$
H_{\mathrm{CR}, T}^{*}(\mathcal{X}) \cong \bigoplus_{v \in V} H_{T}^{*}\left(\mathcal{X}_{v}\right)
$$

and by identifying the untwisted sector with $\mathcal{X}$ itself, we may view $H_{T}^{*}(\mathcal{X})$ as a summand of $H_{\mathrm{CR}, T}^{*}(\mathcal{X})$. There is a natural involution map inv: $I \mathcal{X} \rightarrow I \mathcal{X}$ which sends $(x, g)$ to $\left(x, g^{-1}\right)$. We use this to define a pairing

$$
(\alpha, \beta)_{\mathcal{X}}:=\sum_{v \in V} \int_{\mathcal{X}_{v}} \alpha \cup \operatorname{inv}^{*} \beta
$$

for $\alpha, \beta \in H_{\mathrm{CR}, T}^{*}(\mathcal{X}) \otimes_{R_{T}} S_{T}$, where the integral is defined via localization.

Given elements $\alpha_{1}, \ldots, \alpha_{n}$ of $H_{\mathrm{CR}, T}^{*}(\mathcal{X})$ and integers $a_{1}, \ldots, a_{n} \in \mathbb{Z}_{\geqslant 0}$, we define the GromovWitten invariant as

$$
\left\langle\psi^{a_{1}} \alpha_{1}, \ldots, \psi^{a_{n}} \alpha_{n}\right\rangle_{g, n, d}^{\mathcal{X}}:=\int_{\left[\overline{\mathscr{M}}_{g, n}(\mathcal{X} ; d)\right]^{\mathrm{vir}}} \prod_{i=1}^{n} \psi_{i}^{a_{i}} \mathrm{ev}_{i}^{*}\left(\alpha_{i}\right) .
$$

Here $d \in \mathrm{NE}(X)_{\mathbb{Z}}=\mathrm{NE}(X) \cap H_{2}(|\mathcal{X}| ; \mathbb{Z})$, the space $\overline{\mathscr{M}}_{g, n}(\mathcal{X} ; d)$ is the moduli space of stable maps of degree $d$ from a genus $g$ orbi-curve with $n$ marked points into $\mathcal{X}$, and $[-]^{\text {vir }}$ denotes the virtual class [AGV08]. Finally, $\psi_{i}$ denotes the $\psi$-class at the $i$ th marked point. In the case where $\mathcal{X}$ (and therefore possibly $\overline{\mathscr{M}}_{g, n}(\mathcal{X} ; d)$ ) is not proper, the above integral is defined via the (virtual) Atiyah-Bott localization formula [GP99] and lies in $S_{T}$.

\subsection{Quantum cohomology}

Fix a basis $\left\{\phi_{i}\right\}_{i \in I}$ for $H_{\mathrm{CR}, T}^{*}(\mathcal{X})$. We may express a general point in $H_{\mathrm{CR}, T}^{*}(\mathcal{X})$ as $\boldsymbol{t}=\sum_{i \in I} t^{i} \phi_{i}$. It will be convenient to use the double bracket notation. Given $\alpha_{j} \in H_{\mathrm{CR}, T}^{*}(\mathcal{X})$ and $a_{j} \in \mathbb{Z}_{\geqslant 0}$ as above, define

$$
\left\langle\left\langle\psi^{a_{1}} \alpha_{1}, \ldots, \psi^{a_{n}} \alpha_{n}\right\rangle\right\rangle^{\mathcal{X}}:=\sum_{d \in \mathrm{NE}(X)_{\mathbb{Z}}} \sum_{k=0}^{\infty} \frac{Q^{d}}{k !}\left\langle\psi^{a_{1}} \alpha_{1}, \ldots, \psi^{a_{n}} \alpha_{n}, \boldsymbol{t}, \ldots, \boldsymbol{t}\right\rangle_{0, n+k, d}^{\mathcal{X}},
$$

where on the right-hand side, we declare the degree zero Gromov-Witten invariants with one or two marked points to be zero, since the corresponding moduli spaces are empty. In the above, the variables $Q^{d}$ are so-called Novikov variables, used to guarantee the convergence of the sum.

Definition 3.1. The quantum product $*_{\boldsymbol{t}}: H_{\mathrm{CR}, T}^{*}(\mathcal{X}) \times H_{\mathrm{CR}, T}^{*}(\mathcal{X}) \rightarrow H_{\mathrm{CR}, T}^{*}(\mathcal{X})$ is given by

$$
\left(\alpha *_{\boldsymbol{t}} \beta, \gamma\right)=\langle\langle\alpha, \beta, \gamma\rangle\rangle^{\mathcal{X}} \text {. }
$$

The quantum cohomology ring yields a formal deformation of $H_{\mathrm{CR}, T}^{*}(\mathcal{X})$.

\subsection{The Givental formalism}

This section serves to introduce and explain the origin of the various generating functions which we shall employ. We refer the interested reader to [Giv04] for more information.

Let $\mathcal{H}_{\mathcal{X}}$ denote the vector space $H_{\mathrm{CR}, T}^{*}(\mathcal{X})[[Q]] \otimes_{R_{T}} S_{T}\left(\left(z^{-1}\right)\right)$ endowed with the symplectic pairing

$$
\Omega_{\mathcal{X}}(f, g)=\operatorname{Res}_{z=0}(f(-z), g(z))_{\mathcal{X}} \mathrm{d} z .
$$

The vector space $\mathcal{H}_{\mathcal{X}}$ has a natural polarization given by

$$
\mathcal{H}_{\mathcal{X}}^{+}:=H_{\mathrm{CR}, T}^{*}(\mathcal{X})[[Q]] \otimes_{R_{T}} S_{T}[z] \quad \text { and } \quad \mathcal{H}_{\mathcal{X}}^{-}:=z^{-1} H_{\mathrm{CR}, T}^{*}(\mathcal{X})[[Q]] \otimes_{R_{T}} S_{T}\left[\left[z^{-1}\right]\right] .
$$




\section{QuANTUM COHOMOLOGY OF BLOWUPS}

We obtain Darboux coordinates $\left\{q_{k}^{i}, p_{k, i}\right\}$ with respect to the polarization on $\mathcal{H}_{\mathcal{X}}$ by representing each element of $\mathcal{H}_{\mathcal{X}}$ in the form

$$
\sum_{k \geqslant 0} \sum_{i \in I} q_{k}^{i} \phi_{i} z^{k}+\sum_{k \geqslant 0} \sum_{i \in I} p_{k, i} \phi^{i}(-z)^{-k-1},
$$

where $\left\{\phi^{i}\right\}$ is the dual basis to $\left\{\phi_{i}\right\}$. Define the genus zero generating function $\mathcal{F}_{0}^{\mathcal{X}}: \mathcal{H}_{\mathcal{X}}^{+} \rightarrow S_{T}[[Q]]$ by

$$
\mathcal{F}_{0}^{\mathcal{X}}(\boldsymbol{t}(z)):=\sum_{d \in \mathrm{NE}(X)_{\mathbb{Z}}} \sum_{n=0}^{\infty} \frac{Q^{d}}{n !}\langle\boldsymbol{t}(\psi), \ldots, \boldsymbol{t}(\psi)\rangle_{0, n, d}^{\mathcal{X}},
$$

where $\boldsymbol{t}(z)=\sum_{k \geqslant 0} \sum_{i \in I} t_{k}^{i} \phi_{i} z^{k}$.

One can view $\mathcal{F}_{0}^{\mathcal{X}}$ as the generating function of a Lagrangian subspace $\mathscr{L}_{\mathcal{X}}$ of $\mathcal{H}_{\mathcal{X}}$. Assume that we have chosen a basis such that $\phi_{0}$ denotes the unit in $H_{\mathrm{CR}, T}^{*}(\mathcal{X})$, and make the change of variables (the so-called dilaton shift)

$$
q_{1}^{0}=t_{1}^{0}-1, \quad q_{k}^{i}=t_{k}^{i} \quad \text { for } \quad(k, i) \neq(1,0) .
$$

Then the set

$$
\mathscr{L}_{\mathcal{X}}:=\left\{\mathbf{p}=\mathrm{d}_{\mathbf{q}} \mathcal{F}_{0}^{\mathcal{X}}\right\}
$$

defines a Lagrangian subspace. More explicitly, $\mathscr{L}_{\mathcal{X}}$ contains the points of the form

$$
-z+\boldsymbol{t}(z)+\sum_{d \in \operatorname{NE}(X)_{\mathbb{Z}}} \sum_{\substack{n, a \geqslant 0 \\ i \in I}} \frac{Q^{d}}{n !(-z)^{a+1}}\left\langle\psi^{a} \phi_{i}, \boldsymbol{t}(\psi), \ldots, \boldsymbol{t}(\psi)\right\rangle_{0, n+1}^{\mathcal{X}} \phi^{i} .
$$

Definition 3.2. Given formal parameters $x^{1}, \ldots, x^{r}$, we define an $S_{T}\left[\left[Q, x^{1}, \ldots, x_{r}\right]\right]$-valued point of $\mathscr{L}_{\mathcal{X}}$ to be an element $f(\mathbf{x}) \in \mathcal{H}_{\mathcal{X}}\left[\left[x^{1}, \ldots, x^{r}\right]\right]$ of the form above, such that $\boldsymbol{t}(z) \in$ $\mathcal{H}_{\mathcal{X}}^{+}\left[\left[x^{1}, \ldots, x^{r}\right]\right]$ satisfies $\left.\boldsymbol{t}(z)\right|_{Q=x^{1}=\cdots=x^{r}=0}=0$. We say that the function $f(\mathbf{x})$ lies on $\mathscr{L}_{\mathcal{X}}$.

Due to the string and dilaton equations, and the topological recursion relations [CK99], $\mathscr{L}_{\mathcal{X}}$ takes a special form, known as an overruled Lagrangian cone. In particular, $\mathscr{L}_{\mathcal{X}}$ is a cone satisfying the condition that for all $f \in \mathcal{H}_{\mathcal{X}}$,

$$
\mathscr{L}_{\mathcal{X}} \cap L_{f}=z L_{f}
$$

where $L_{f}$ is the tangent space to $\mathscr{L}_{\mathcal{X}}$ at $f$. Equation (3.1) justifies the term overruled, as each tangent space $L_{f}$ is filtered by powers of $z$ :

$$
L_{f} \supset z L_{f} \supset z^{2} L_{f} \supset \cdots
$$

and $\mathscr{L}_{\mathcal{X}}$ itself is ruled by the various $z L_{f}$. The codimension of $z L_{f}$ in $L_{f}$ is equal to $\operatorname{dim}\left(H_{\mathrm{CR}, T}^{*}(\mathcal{X})\right)$. The upshot of this is that a generic slice of $\mathscr{L}_{\mathcal{X}}$ parametrized by $H_{\mathrm{CR}, T}^{*}(\mathcal{X})$, that is,

$$
\left\{f(\boldsymbol{t}) \mid \boldsymbol{t} \in H_{\mathrm{CR}, T}^{*}(\mathcal{X})\right\} \subset \mathscr{L}_{\mathcal{X}},
$$

will be transverse to the ruling. Given such a slice, we can reconstruct $\mathscr{L}_{\mathcal{X}}$ as

$$
\mathscr{L}_{\mathcal{X}}=\left\{z L_{f(\boldsymbol{t})} \mid \boldsymbol{t} \in H_{\mathrm{CR}, T}^{*}(\mathcal{X})\right\} .
$$

The most well-known slice of $\mathscr{L}_{\mathcal{X}}$ is given by Givental's $J$-function [Giv04]. 


\section{P. Acosta And M. Shoemaker}

Definition 3.3. The Givental $J$-function of $\mathcal{X}$ is the cohomology-valued generating function of genus zero Gromov-Witten invariants given by

$$
J^{\mathcal{X}}(\boldsymbol{t},-z)=-z+\boldsymbol{t}+\sum_{i \in I}\left\langle\left\langle\frac{\phi_{i}}{z-\psi_{1}}\right\rangle\right\rangle^{\mathcal{X}} \phi^{i},
$$

where the expression $1 /(z-\psi)$ is shorthand for the corresponding expansion in $1 / z$.

Due to the divisor equation, the $J$-function also makes sense in the specialization $Q=1$. Thus, we can safely forget the Novikov variables. Note that $J^{\mathcal{X}}(\boldsymbol{t},-z)=-z+\boldsymbol{t} \oplus \mathcal{H}_{\mathcal{X}}^{-} \cap \mathscr{L}_{\mathcal{X}}$. One can easily check that the $J$-function is transverse to the ruling and therefore determines all of $\mathscr{L}_{\mathcal{X}}$.

Remark 3.4. In many situations, one can use mirror symmetry to find another function $I(\mathbf{x}, z)$, called an $I$-function, which lies on $\mathscr{L}_{\mathcal{X}}$ in the sense of Definition 3.2. We say that $I(\mathbf{x}, z)$ is a big $I$-function if its image is transverse to the ruling. In what follows, we will work with big $I$-functions, as it is generally difficult to obtain a closed form for the $J$-function. These functions, while defined combinatorially in terms of toric data, fully determine the $J$-function and thus the Lagrangian cone through a process of Birkhoff factorization (see [CIJ14, Theorem 5.15]).

\section{Weighted blowups of $\left[\mathbb{C}^{N} / G\right]$}

In this section, we introduce the specific spaces of interest and describe the corresponding $I$ functions.

We will restrict ourselves to the particular blowups of relevance to the Landau-Ginzburg correspondences of Section 6. Although the proof holds for more general toric blowups with minimal modification, this simplifies the notation and exposition.

In particular, we consider birational spaces $\mathcal{Y} \rightarrow \mathcal{X}$ where $\mathcal{X}$ is a quotient stack of the form $\left[\mathbb{C}^{N} / G\right]$ and $\mathcal{Y}$ is a line bundle over weighted projective space obtained as a blowup of $\mathcal{X}$. In anticipation of our application to FJRW theory, we require that $G$ arise as a subgroup of the diagonal automorphisms of the Fermat polynomial

$$
W=X_{1}^{d / c_{1}}+\cdots+X_{N}^{d / c_{N}}
$$

where $\operatorname{gcd}\left(c_{1}, \ldots, c_{N}\right)=1$. We assume that $G$ contains the distinguished automorphism

$$
\mathfrak{j}=\exp \left(2 \pi i \operatorname{diag}\left(q_{1}, \ldots, q_{N}\right)\right),
$$

where the $q_{j}=c_{j} / d$ are the fractional weights of $W$.

\subsection{A toric description of the spaces}

Let $\mathbf{N} \cong \mathbb{Z}^{N}$ be a lattice, and let $\Sigma \subset \mathbf{N} \otimes \mathbb{R}$ denote a fan such that $X_{\Sigma}$ is isomorphic to $\mathcal{X}=\left[\mathbb{C}^{N} / G\right]$. The fan $\Sigma$ contains a single maximal cone with generators $b_{1}, \ldots, b_{N}$, and $G \cong$ $\mathbf{N} /\left\langle b_{1}, \ldots, b_{N}\right\rangle$. Let

$$
\operatorname{Box}(\Sigma)=\left\{b^{\prime}=\sum_{i=1}^{N} m_{i} b_{i} \in \mathbf{N} \mid m_{i} \in \mathbb{Q} \cap[0,1) \text { for } 1 \leqslant i \leqslant N\right\} .
$$

Note that the elements of $\operatorname{Box}(\Sigma)$ are in bijection with those in $G$ and therefore index components of $I \mathcal{X}$.

The element $\mathfrak{j} \in G$ corresponds to the point $b^{\prime}=\sum_{i=1}^{N} q_{i} b_{i} \in \operatorname{Box}(\Sigma)$. Let $\Sigma^{\prime}$ denote the star subdivision of $\Sigma$ obtained by adding the ray generated by $b^{\prime}$. Then $\mathcal{Y}:=X_{\Sigma^{\prime}}$ is isomorphic to 


\section{QUANTUM COHOMOLOGY OF BLOWUPS}

the total space of the vector bundle $\mathscr{O}_{\mathbb{P}(G)}(-d)$ over the stack $\mathbb{P}(G):=\left[\mathbb{P}\left(c_{1}, \ldots, c_{N}\right) / \bar{G}\right]$, where $\bar{G}=G /\langle\mathfrak{j}\rangle$. Thus, $\mathcal{Y}$ gives a partial resolution of the coarse space of $\mathcal{X}$.

One can easily check that the discrepancy of the toric morphism $|f|: \mathcal{Y} \rightarrow|\mathcal{X}|$ is given by $\operatorname{disc}(|f|)=\sum_{j=1}^{N} q_{j}-1$. This will play an important role in what follows.

We endow $\mathcal{X}$ and $\mathcal{Y}$ with compatible torus actions. Let $T \cong \mathbb{C}^{*}$ act on the coordinates of $\mathcal{X}=\left[\mathbb{C}^{N} / G\right]$ with weights $-c_{1}, \ldots,-c_{N}$. On $\mathcal{Y}=\operatorname{Tot}\left(\mathscr{O}_{\mathbb{P}(G)}(-d)\right)$, this corresponds to a trivial action on the base $\mathbb{P}(G)$ with a nontrivial action of weight $d$ in the fiber direction. We let $\lambda$ denote the equivariant parameter of our torus action in $H_{\mathrm{CR}, T}^{*}(\mathcal{X})$ and $H_{\mathrm{CR}, T}^{*}(\mathcal{Y})$.

Notation 4.1. For $g \in G$, we may express the action of $g$ on $\mathbb{C}^{N}$ by

$$
\exp \left(2 \pi i \operatorname{diag}\left(m_{1}(g), \ldots, m_{N}(g)\right)\right),
$$

where $m_{1}(g), \ldots, m_{N}(g) \in \mathbb{Q} \cap[0,1)$. We call $m_{j}(g)$ for $1 \leqslant j \leqslant N$ the multiplicities of $g$. The age of $g$ is defined as $\sum_{j=1}^{N} m_{j}(g)$.

Note that for any element $g \in G$, the fact that $G$ preserves the polynomial $W$ implies that $m_{j}(g)$ is a multiple of $q_{j}$ for $1 \leqslant j \leqslant N$. This implies that $G$ splits as $G \cong\langle j\rangle \oplus \bar{G}$. Let us once and for all fix a splitting of $G$. Choose generators $g_{1}, \ldots, g_{k}$ of $\bar{G}$ such that each $g_{i}$ fixes the first coordinate of $\mathbb{C}^{N}$ (this can be done without loss of generality due to the restrictions on the multiplicities $\left.m_{1}\left(g_{i}\right)\right)$ and $G \cong\langle\mathfrak{j}\rangle \oplus\left\langle g_{1}\right\rangle \oplus \cdots \oplus\left\langle g_{k}\right\rangle$.

Remark 4.2. The condition that the generators $g_{1}, \ldots, g_{k}$ each fix the first coordinate is not necessary to prove the correspondence for toric blowups. It will be used in the last section to simplify the differential equations satisfied by the two $I$-functions.

Notation 4.3. We let $\bar{G}$ denote the group generated by $g_{1}, \ldots, g_{k}$ from above.

\section{$4.2 I$-functions}

4.2.1 The $I$-function of $\mathcal{X}$. The inertia stack $I \mathcal{X}$ is a disjoint union of components $\mathcal{X}_{g}$ indexed by $g \in G$. There is a natural choice of basis for the equivariant cohomology of $\mathcal{X}$ given by $\left\{\mathbb{1}_{g}\right\}_{g \in G}$, where $\mathbb{1}_{g}$ is the fundamental class of $\mathcal{X}_{g}$. By abuse of notation, we will also use $\mathbb{1}_{g}$ to denote the fundamental class of the $g$ th component of the inertia stack of $\mathrm{B} G$.

Notation 4.4. It will be convenient to separate components of $I \mathcal{X}$ according to $\bar{G}$-cosets. Namely,

$$
I \mathcal{X}=\bigcup_{g \in \bar{G}} \bigcup_{0 \leqslant k \leqslant d-1} \mathcal{X}_{\mathfrak{j}^{k} g} .
$$

Let $H_{g}^{*}(\mathcal{X}):=H_{T}^{*}\left(\bigcup_{0 \leqslant k \leqslant d-1} \mathcal{X}_{\mathrm{j}^{k} g}\right)$ denote the corresponding subspace in $H_{\mathrm{CR}, T}^{*}(\mathcal{X})$. We see that this has dimension $d$.

Let $t^{g}$ denote the dual coordinate of $\mathbb{1}_{g}$ for $g \in \bar{G}$, and let $t$ denote the dual coordinate of $\mathfrak{j}$.

We consider the $J$-function of $\mathrm{B} G$, where the domain has been restricted to the span of $\left\{\mathbb{1}_{\mathfrak{j}}\right\} \cup\left\{\mathbb{1}_{g}\right\}_{g \in \bar{G}}$. A simple computation involving $\psi$-classes on $\overline{\mathscr{M}}_{0, n}$ allows us to obtain an explicit formula (see [LPS16, Lemmas 5.2 and 7.2]):

$$
J^{\mathrm{B} G}(t, \boldsymbol{t}, z)=z \sum_{\mathbf{k} \in\left(\mathbb{Z}_{\geqslant 0}\right)^{\bar{G}}} \prod_{g \in \bar{G}} \frac{\left(t^{g}\right)^{k_{g}}}{z^{k_{g}} k_{g} !} \sum_{k_{0} \geqslant 0} \frac{t^{k_{0}}}{z^{k_{0}} k_{0} !} \mathbb{1}_{\mathfrak{j}^{k_{0}}} \prod_{g} g^{k_{g}} .
$$




\section{P. Acosta And M. Shoemaker}

Using twisted theory technology, one may alter $J^{\mathrm{B} G}(t, \boldsymbol{t}, z)$ by a hypergeometric modification (see [CCIT09]) to obtain an $I$-function, $I^{\mathcal{X}}(t, \boldsymbol{t}, z)$ in the sense of Remark 3.4. Let $a(\mathbf{k})^{j}=$ $\sum_{g \in \bar{G}} k_{g} m_{j}(g)$. Define the modification factor

$$
M\left(k_{0}, \mathbf{k}\right):=\prod_{j=1}^{N} \prod_{l=0}^{\left\lfloor k_{0} q_{j}+a(\mathbf{k})^{j}\right\rfloor-1}\left(-c_{j} \lambda-\left(\left\langle k_{0} q_{j}+a(\mathbf{k})^{j}\right\rangle+l\right) z\right)
$$

where $\langle-\rangle$ denotes the fractional part. Then $I^{\mathcal{X}}(t, t, z)$ is defined as

$$
I^{\mathcal{X}}(t, \boldsymbol{t}, z)=z t^{d \lambda / z} \sum_{\mathbf{k} \in\left(\mathbb{Z}_{\geqslant 0}\right)} \prod_{\bar{G}} \frac{\left(t^{g}\right)^{k_{g}}}{z^{k_{g}} k_{g} !} \sum_{k_{0} \geqslant 0} \frac{M\left(k_{0}, \mathbf{k}\right) t^{k_{0}}}{z^{k_{0}} k_{0} !} \mathbb{1}_{\mathfrak{j}^{k_{0}}} \prod_{g} g^{k_{g}} .
$$

The above modification factor is explained in [CCIT09], where it is proven that $I^{\mathcal{X}}(t, \boldsymbol{t}, z)$ is a (big) $I$-function for $\mathcal{X}$. Using Gamma functions, this simplifies to

$$
\begin{aligned}
I^{\mathcal{X}}(t, \boldsymbol{t}, z)= & z t^{d \lambda / z} \sum_{\mathbf{k} \in\left(\mathbb{Z}_{\geqslant 0}\right) \bar{G}} \prod_{g \in \bar{G}} \frac{\left(t^{g}\right)^{k_{g}} z^{(\operatorname{age}(g)-1) k_{g}}}{k_{g} !} \sum_{k_{0} \geqslant 0} \frac{t^{k_{0}} z^{k_{0}\left(\sum_{j} q_{j}-1\right)}}{z^{\sum_{j}\left\langle k_{0} q_{j}+a(\mathbf{k})^{j}\right\rangle} k_{0} !} \\
& \times \prod_{j=1}^{N} \frac{\Gamma\left(1-c_{j} \frac{\lambda}{z}-\left\langle k_{0} q_{j}+a(\mathbf{k})^{j}\right\rangle\right)}{\Gamma\left(1-c_{j} \frac{\lambda}{z}-k_{0} q_{j}-a(\mathbf{k})^{j}\right)} \mathbb{1}_{\mathfrak{j}^{k_{0}}} \prod_{g} g^{k_{g}} \cdot
\end{aligned}
$$

4.2.2 The $I$-function of $\mathcal{Y}$. The components of the inertia stack $I \mathcal{Y}$ are indexed by $\{g\}_{g \in G}$. This follows from the facts that, first, each $c_{j}$ divides $d$ (and so components of $I \mathbb{P}\left(c_{1}, \ldots, c_{N}\right)$ correspond to powers of $\mathfrak{j}$ ) and, second, $G$ splits as $\langle\mathfrak{j}\rangle \oplus \bar{G}$. Here, the component $\mathcal{Y}_{g}$ of $I \mathcal{Y}$ is identified with the closed subset of $\mathcal{Y}$ obtained by setting $x_{j}=0$ for all coordinates not fixed by $g$ (that is, $m_{j}(g) \neq 0$ ). An equivariant basis for the Chen-Ruan cohomology of $\mathcal{Y}$ is given by

$$
\bigcup_{\left\{g \in G \mid \operatorname{dim}\left(\left(\mathbb{C}^{N}\right)^{g}\right)>0\right\}}\left\{\tilde{\mathbb{1}}_{g}, \tilde{\mathbb{1}}_{g} H, \ldots, \tilde{\mathbb{1}}_{g} H^{\left(\operatorname{dim}\left(\left(\mathbb{C}^{N}\right)^{g}\right)-1\right)}\right\},
$$

where $\tilde{\mathbb{1}}_{g}$ is the fundamental class of $\mathcal{Y}_{g}$ and $\tilde{\mathbb{1}}_{g} H^{k}$ denotes the pullback of the $k$ th power of the hyperplane class from the coarse space of $\mathcal{Y}_{g}$. In what follows, it will simplify our formulas to also sometimes write $\tilde{\mathbb{1}}_{g}$ when the action of $g$ on $\mathbb{C}^{N}$ fixes only the origin (and thus $\mathcal{Y}_{g}$ is empty) we follow the convention $\tilde{\mathbb{1}}_{g}=0$ in this case.

Notation 4.5. We may also separate components of $I \mathcal{Y}$ according to $\bar{G}$-cosets,

$$
I \mathcal{Y}=\bigcup_{g \in \bar{G}} \bigcup_{0 \leqslant k \leqslant d-1} \mathcal{Y}_{j^{k} g}
$$

One may check that the corresponding subspace $H_{g}^{*}(\mathcal{Y}):=H_{T}^{*}\left(\bigcup_{0 \leqslant k \leqslant d-1} \mathcal{Y}_{j^{k} g}\right)$ has dimension equal to $c_{1}+\cdots+c_{N}$.

We will also let $t^{g}$ denote the dual coordinate of $\tilde{\mathbb{1}}_{g}$ for $g \in G$ and let $q$ denote the exponential of the dual coordinate to $H$. 


\section{QUANTUM COHOMOLOGY OF BLOWUPS}

An $I$-function for toric stacks is given in [CCIT15]. Again using Gamma functions, a (big) $I$-function for $\mathcal{Y}$ takes the form

$$
\begin{aligned}
I^{\mathcal{Y}}(q, \boldsymbol{t}, z)= & z q^{H / z} \sum_{\mathbf{k} \in\left(\mathbb{Z}_{\geqslant 0}\right)} \prod_{\bar{G}^{\bar{G}} \in \bar{G}} \frac{\left(t^{g}\right)^{k_{g}} z^{(\operatorname{age}(g)-1) k_{g}}}{k_{g} !} \sum_{k_{0} \geqslant 0} \frac{q^{k_{0} / d}}{z^{k_{0}\left(\sum_{j} q_{j}-1\right)+\sum_{j}\left\langle k_{0} q_{j}-a(\mathbf{k})^{j}\right\rangle}} \\
& \times \frac{\Gamma\left(1-\frac{d(\lambda+H)}{z}\right)}{\Gamma\left(1-k_{0}-\frac{d(\lambda+H)}{z}\right)} \prod_{j=1}^{N} \frac{\Gamma\left(1+c_{j} H / z-\left\langle-k_{0} q_{j}+a(\mathbf{k})^{j}\right\rangle\right)}{\Gamma\left(1+c_{j} H / z+k_{0} q_{j}-a(\mathbf{k})^{j}\right)} \tilde{\mathbb{1}}_{\mathfrak{j}-k_{0}} \prod_{g} g^{k_{g}} .
\end{aligned}
$$

Remark 4.6. Note that we have applied the specialization of the Novikov variable: $Q=1$. There is no loss of information here, as one can recover the Novikov variable in $I^{\mathcal{Y}}(q, \boldsymbol{t}, z)$ via $q^{k_{0} / d} \mapsto Q^{k_{0}} q^{k_{0} / d}$. However, the specialization simplifies our correspondence between $I^{\mathcal{X}}$ and $I^{\mathcal{Y}}$.

\section{The correspondence for toric blowups}

\subsection{Asymptotic correspondence for positive discrepancy}

In this section, we will state a correspondence of genus zero Gromov-Witten theories for the case where $|f|: \mathcal{Y} \rightarrow|\mathcal{X}|$ has positive discrepancy. In Section 5.5, we will discuss the other case. Recall that the discrepancy of $|f|$ is equal to $\sum_{j=1}^{N} q_{j}-1$. To simplify the notation slightly, we will work with the quantity $r=d \cdot \operatorname{disc}(|f|)$,

$$
r:=\sum_{j=1}^{N} c_{j}-d .
$$

The idea behind the asymptotic correspondence of genus zero theories is to obtain the genus zero Gromov-Witten invariants of $\mathcal{X}$ using the information provided by the genus zero GromovWitten invariants of $\mathcal{Y}$. To be more specific, we show that the $I$-function of $\mathcal{X}$ (and therefore its $J$-function) is completely determined by the $I$-function of $\mathcal{Y}$ through power series asymptotic expansion.

Theorem 5.1. Assume $\operatorname{disc}(|f|)>0$. There exists a unique linear transformation

$$
L: H_{\mathrm{CR}, T}^{*}(\mathcal{Y}) \longrightarrow H_{\mathrm{CR}, T}^{*}(\mathcal{X})
$$

such that

$$
L \cdot I^{\mathcal{Y}}(q, \boldsymbol{t}, z=1) \sim I^{\mathcal{X}}\left(t=q^{-1 / d}, \boldsymbol{t}, z=1\right) \quad \text { as } q \rightarrow \infty .
$$

Remark 5.2. One can reinstate the $z$-parameter into the $I$-functions and prove an analogous version of the above theorem, where the linear transformation $L(z)$ is now viewed as a map from $\mathcal{H}_{\mathcal{Y}}$ to $\mathcal{H}_{\mathcal{X}}$ [AS16]. We expect that $L(z)$ will be compatible with the symplectic structures as well as the natural grading in $\mathcal{H}_{\mathcal{Y}}$ and $\mathcal{H}_{\mathcal{X}}$. There is reason to also expect that the quantization $\widehat{L}(z)$ relates the higher-genus Gromov-Witten invariants of $\mathcal{Y}$ and $\mathcal{X}$ (see, for instance, [CI14] and [CR13]). We plan to investigate these questions in future work.

Theorem 5.1 has the following consequence.

Corollary 5.3. If $\operatorname{disc}(|f|)>0$, then the genus zero Gromov-Witten invariants of $\mathcal{X}$ are completely determined by the genus zero Gromov-Witten invariants of $\mathcal{Y}$. 


\section{P. Acosta And M. Shoemaker}

Proof. First, note that $I^{\mathcal{Y}}$ may be viewed as a generating function of genus zero Gromov-Witten invariants. Next, note that $I^{\mathcal{X}}\left(t=q^{-1 / d}, \boldsymbol{t}, z=1\right)$ is the power series asymptotic expansion of $L \cdot I^{\mathcal{Y}}(q, \boldsymbol{t}, z=1)$, and it is therefore uniquely determined by $I^{\mathcal{Y}}$. We may recover $I^{\mathcal{X}}(t=$ $\left.q^{-1 / d}, \boldsymbol{t}, z\right)$ from $I^{\mathcal{X}}\left(t=q^{-1 / d}, \boldsymbol{t}, 1\right)$ by means of the relations described in Section 5.2 below. Last, the Givental $J$-function of $\mathcal{X}$ (and therefore, its genus zero theory) can be obtained from $I^{\mathcal{X}}$ via Birkhoff factorization (see Remark 3.4).

The proof that follows relies heavily on the recursive structure of the functions $I^{\mathcal{Y}}$ and $I^{\mathcal{X}}$. In particular, we take advantage of an identification of the differential equations satisfied by the respective $I$-functions to relate the functions themselves. This is part of the larger theory of GKZ systems described by Gelfand-Kapranov-Zelevinsky in [GZK89] and Adolphson in [Ado94], although in what follows, the relevant differential equations can easily be checked by hand.

Notation 5.4. In order to simplify the computations in the subsequent sections, we introduction the following notation. Define

$$
\begin{aligned}
I_{\mathbf{k}}^{\mathcal{Y}}(q, z):= & z \sum_{k_{0} \geqslant 0} \frac{q^{k_{0} / d+H / z}}{z^{k_{0} r / d+\sum_{j}\left\langle k_{0} q_{j}-a(\mathbf{k})^{j}\right\rangle}} \frac{\Gamma(1-d(\lambda+H) / z)}{\Gamma\left(1-k_{0}-d(\lambda+H) / z\right)} \\
& \times \prod_{j=1}^{N} \frac{\Gamma\left(1+c_{j} H / z-\left\langle-k_{0} q_{j}+a(\mathbf{k})^{j}\right\rangle\right)}{\Gamma\left(1+c_{j} H / z+k_{0} q_{j}-a(\mathbf{k})^{j}\right)} \tilde{\mathbb{1}}_{\mathfrak{j}^{-k_{0}} \prod_{g} g^{k_{g}}}
\end{aligned}
$$

and

$$
I_{\mathbf{k}}^{\mathcal{X}}(t, z):=z \sum_{k_{0} \geqslant 0} \frac{t^{k_{0}+d \lambda / z} z^{k_{0} r / d}}{z^{\sum_{j}\left\langle k_{0} q_{j}+a(\mathbf{k})^{j}\right\rangle} k_{0} !} \prod_{j=1}^{N} \frac{\Gamma\left(1-c_{j} \lambda / z-\left\langle k_{0} q_{j}+a(\mathbf{k})^{j}\right\rangle\right)}{\Gamma\left(1-c_{j} \lambda / z-k_{0} q_{j}-a(\mathbf{k})^{j}\right)} \mathbb{1}_{\mathfrak{j}^{k_{0}}} \prod_{g} g^{k_{g}} .
$$

Then, the $I$-functions of $\mathcal{Y}$ and $\mathcal{X}$ can be, respectively, written as

$$
\begin{aligned}
& I^{\mathcal{Y}}(q, \boldsymbol{t}, z)=\sum_{\mathbf{k} \in\left(\mathbb{Z}_{\geqslant 0}\right)^{\bar{G}}} \prod_{g \in \bar{G}} \frac{\left(t^{g}\right)^{k_{g}} z^{(\operatorname{age}(g)-1) k_{g}}}{k_{g} !} I_{\mathbf{k}}^{\mathcal{Y}}(q, z), \\
& I^{\mathcal{X}}(t, \boldsymbol{t}, z)=\sum_{\mathbf{k} \in\left(\mathbb{Z}_{\geqslant}\right)} \prod_{\bar{G}} \frac{\left(t^{g}\right)^{k_{g}} z^{(\operatorname{age}(g)-1) k_{g}}}{k_{g} !} I_{\mathbf{k}}^{\mathcal{X}}(t, z) .
\end{aligned}
$$

\subsection{Setting $z$ equal to 1}

In what follows, we will set $z=1$ in the $I$-functions for computational convenience. It is worth noting that we can recover the original $I$-functions by the following procedure. Define a grading operator $\mathbf{G r}$ by

$$
\begin{aligned}
& \mathbf{G r}(H):=H, \quad \mathbf{G r}(\lambda):=\lambda, \quad \mathbf{G r}\left(t^{g}\right):=(1-\operatorname{age}(g)) t^{g},
\end{aligned}
$$

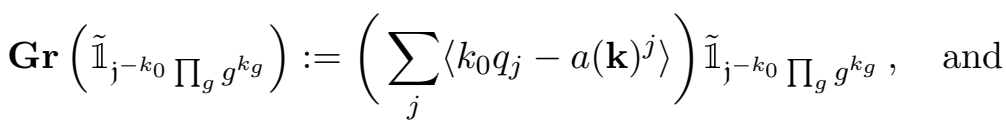

$$
\begin{aligned}
& \operatorname{Gr}\left(\mathbb{1}_{\mathfrak{j}^{k_{0}} \prod_{g} g^{k_{g}}}\right):=\left(\sum_{j}\left\langle k_{0} q_{j}+a(\mathbf{k})^{j}\right\rangle\right) \mathbb{1}_{\mathfrak{j}^{k_{0}} \prod_{g} g^{k_{g}}} .
\end{aligned}
$$




\section{QUANTUM COHOMOLOGY OF BLOWUPS}

We then have the following relations that allow us to restore the powers of $z$ to the $I$-functions:

$$
\begin{aligned}
& I^{\mathcal{Y}}(q, \boldsymbol{t}, z)=z^{1-\mathbf{G r}} z^{r H} I^{\mathcal{Y}}\left(q / z^{r}, \boldsymbol{t}, 1\right) \text { and } \\
& I^{\mathcal{X}}(t, \boldsymbol{t}, z)=z^{1-\mathbf{G r}} z^{-r \lambda} I^{\mathcal{X}}\left(t z^{r / d}, \boldsymbol{t}, 1\right) .
\end{aligned}
$$

\subsection{The regularized $I$-function}

We define the regularized I-function of $\left[\mathbb{C}^{N} / G\right]$ by

$$
I_{\mathbf{k}}^{\mathrm{reg}}(\tau):=\sum_{k_{0} \geqslant 0} \frac{\tau^{r\left(k_{0} / d+\lambda\right)}}{\Gamma\left(1+r\left(k_{0} / d+\lambda\right)\right) k_{0} !} \prod_{j=1}^{N} \frac{\Gamma\left(1-c_{j} \lambda-\left\langle k_{0} q_{j}+a(\mathbf{k})^{j}\right\rangle\right)}{\left.\Gamma\left(1-c_{j} \lambda-k_{0} q_{j}-a(\mathbf{k})^{j}\right)\right)} \mathbb{1}_{\mathfrak{j}^{k_{0}}} \prod_{g} g^{k_{g}} .
$$

Using the ratio test, it is easy to see that this series defines a holomorphic function in a disk of radius $\left(r^{r} d^{d} \prod_{j=1}^{N} c_{j}^{-c_{j}}\right)^{1 / r}$ centered at $\tau=0$. A simple computation reveals that $I_{\mathbf{k}}^{\text {reg }}(\tau)$ satisfies the following regularized Picard-Fuchs equation:

$$
\left[\tau^{r} \prod_{j=1}^{N} \prod_{l=0}^{c_{j}-1}\left(-\frac{c_{j}}{r} \tau \frac{\mathrm{d}}{\mathrm{d} \tau}-l-a(\mathbf{k})^{j}\right)-\prod_{l=0}^{d-1}\left(\frac{d}{r} \tau \frac{\mathrm{d}}{\mathrm{d} \tau}-d \lambda-l\right) \prod_{l=0}^{r-1}\left(\tau \frac{\mathrm{d}}{\mathrm{d} \tau}-l\right)\right] I_{\mathbf{k}}^{\mathrm{reg}}(\tau)=0 .
$$

This equation has singular points at $\tau=0, \infty$ and at $\tau$ satisfying $(-\tau / r)^{r}=(-d)^{d} \prod_{j=1}^{N} c_{j}^{-c_{j}}$. All of these points are regular singularities. This means that $I_{\mathbf{k}}^{\mathrm{reg}}(\tau)$ can be analytically continued to $\tau=\infty$ along any ray that avoids these singularities.

\subsection{Proof of Theorem 5.1}

The idea behind the proof of Theorem 5.1 consists in constructing a holomorphic function that satisfies the Picard-Fuchs equation of $\mathcal{Y}$ and whose asymptotic expansion is given by the $I$ function of $\mathcal{X}$. To construct this function, we use the following Laplace integral of the regularized I-function:

$$
\mathbb{I}_{\mathbf{k}}(u):=u \int_{0}^{\infty} e^{-u \tau} I_{\mathbf{k}}^{\mathrm{reg}}(\tau) \mathrm{d} \tau
$$

where the ray of integration lies in $0<\arg (x)<\pi / \sum c_{j}$ in order to avoid the singular points of $I_{\mathbf{k}}^{\mathrm{reg}}(\tau)$. This integral is well defined, as shown in [MSH10, Chapter 2].

To compute the asymptotic expansion of $\mathbb{I}_{\mathbf{k}}(u)$, we make use of Watson's lemma. The setup is as follows:

Let $\phi(x)$ be a complex-valued function of the form $\phi(x)=x^{\sigma} g(x)$, where

(i) there exist angles $\alpha$ and $\beta$ such that $\phi(x)$ is defined for $x \neq 0$ with $-\pi / 2 \leqslant \alpha<\arg (x)<$ $\beta \leqslant \pi_{2}$

(ii) for some positive number $b$, we have $|\phi(x)|<e^{b|x|}$ for $|x|$ sufficiently large on this domain;

(iii) $g(x)$ is infinitely differentiable in a neighborhood of $x=0$ and $g(0) \neq 0$;

(iv) $\mathfrak{R e}(\sigma)>-1$;

then the integral $u \int_{0}^{\infty} e^{-u x} \phi(x) \mathrm{d} x$ is well defined for any $u$ in the region $-\pi / 2-\beta<\arg (u)<$ $\pi / 2-\alpha$. Here, the path of integration is taken to be any ray in $\alpha<\arg (x)<\beta$. The integral is independent of this choice of path.

LEMma 5.5 (Watson's lemma). In the situation above, the following asymptotic expansion holds:

$$
u \int_{0}^{\infty} e^{-u x} \phi(x) \mathrm{d} x \sim \sum_{n=0}^{\infty} \frac{g^{(n)}(0) \Gamma(1+\lambda+n)}{n ! u^{\lambda+n}}
$$




\section{P. Acosta And M. Shoemaker}

as $u \rightarrow \infty$ along any ray in the region $-\pi / 2-\beta<\arg (u)<\pi / 2-\alpha$.

One proof of this lemma is given in [Mil06, Proposition 2.1]. Although the conditions on $\phi(x)$ are slightly different in [Mil06] than those stated above, in fact the same proof implies the result in this case as well (see [Mil06, Section 2.3] or [Tem13] for the version given above).

As a consequence of Watson's lemma, the asymptotic expansion of $\mathbb{I}_{\mathbf{k}}(u)$ is given by

$$
\mathbb{I}_{\mathbf{k}}(u) \sim \sum_{k_{0} \geqslant 0} \frac{1}{u^{r\left(k_{0} / d+\lambda\right)} k_{0} !} \prod_{j=1}^{N} \frac{\Gamma\left(1-c_{j} \lambda-\left\langle k_{0} q_{j}+a(\mathbf{k})^{j}\right\rangle\right)}{\left.\Gamma\left(1-c_{j} \lambda-k_{0} q_{j}-a(\mathbf{k})^{j}\right)\right)} \mathbb{1}_{\mathfrak{j}^{k_{0}}} \prod_{g} g^{k_{g}}
$$

as $u \rightarrow \infty$ in the region $|\arg (u)|<\pi / 2$. It follows from this that

$$
\mathbb{I}_{\mathbf{k}}\left(u=q^{1 / r}\right) \sim I_{\mathbf{k}}^{\mathcal{X}}\left(t=q^{-1 / d}, z=1\right)
$$

as $q \rightarrow \infty$.

Define $\mathbb{I}(u)$ to be

$$
\mathbb{I}(u):=\sum_{\mathbf{k} \in\left(\mathbb{Z}_{\geqslant 0}\right)^{\bar{G}}} \prod_{g \in \bar{G}} \frac{\left(t^{g}\right)^{k_{g}}}{k_{g} !} \mathbb{I}_{\mathbf{k}}(u) .
$$

The following lemma and corollaries show that $\mathbb{I}$ satisfies the Picard-Fuchs equation of $\mathcal{Y}$.

Lemma 5.6. The function $\mathbb{I}_{\mathbf{k}}(u)$ satisfies the following differential equation:

$$
\left[\prod_{j=1}^{N} \prod_{l=0}^{c_{j}-1}\left(\frac{c_{j}}{r} u \frac{\mathrm{d}}{\mathrm{d} u}-l-a(\mathbf{k})^{j}\right)-u^{r} \prod_{l=0}^{d-1}\left(-\frac{d}{r} u \frac{\mathrm{d}}{\mathrm{d} u}-d \lambda-l\right)\right] \mathbb{I}_{\mathbf{k}}(u)=0 .
$$

Furthermore, given $\mathbf{k} \in\left(\mathbb{Z}_{\geqslant 0}\right)^{\bar{G}}$, let $\mathbf{g}=\prod_{g \in \bar{G}} g^{k_{g}}$. Then for all $1 \leqslant j \leqslant N$, we have $a(\mathbf{k})^{j}-$ $m_{j}(\mathbf{g})=M_{j}$ for some integer $M_{j} \geqslant 0$ and

$$
\prod_{j=1}^{N} \prod_{l=0}^{M_{j}-1}\left(\frac{c_{j}}{r} u \frac{\mathrm{d}}{\mathrm{d} u}-l-m_{j}(\mathbf{g})\right) \mathbb{I}_{\mathbf{k}(\mathbf{g})}(u)=\mathbb{I}_{\mathbf{k}}(u)
$$

where $\mathbf{k}(\mathbf{g})$ is defined by

$$
\mathbf{k}(\mathbf{g}): g \mapsto \begin{cases}1 & \text { if } g=\mathbf{g} \\ 0 & \text { otherwise }\end{cases}
$$

Proof. Let $f(\tau)$ be holomorphic in some region of the complex plane containing a ray on which we can define the Laplace transform of $f$. Moreover, let $f(0)=0$, and define $F(u):=$ $u \int_{0}^{\infty} e^{-u \tau} f(\tau) \mathrm{d} \tau$. Using the properties of the Laplace transform, it is easy to see that

$$
\begin{aligned}
u \mathcal{L}\left(\alpha \tau \frac{\mathrm{d}}{\mathrm{d} \tau} f(\tau)+\beta f(\tau)\right) & =-\alpha u \frac{\mathrm{d}}{\mathrm{d} u}\left(\mathcal{L}\left(\frac{\mathrm{d}}{\mathrm{d} \tau} f(\tau)\right)\right)+\beta u \mathcal{L}(f(\tau)) \\
& =-\alpha u \frac{\mathrm{d}}{\mathrm{d} u}(u \mathcal{L}(f(\tau))-f(0))+\beta u \mathcal{L}(f(\tau)) \\
& =\left(-\alpha u \frac{\mathrm{d}}{\mathrm{d} u}+\beta\right) F(u),
\end{aligned}
$$

where $\alpha$ and $\beta$ are arbitrary complex numbers. 


\section{QuANTUM COHOMOLOGY OF BLOWUPS}

Now, note that equation (5.2) can be rewritten as

$$
\left[\prod_{j=1}^{N} \prod_{l=0}^{c_{j}-1}\left(-\frac{c_{j}}{r} \tau \frac{\mathrm{d}}{\mathrm{d} \tau}-l-a(\mathbf{k})^{j}\right)-\left(\frac{\mathrm{d}}{\mathrm{d} \tau}\right)^{r} \prod_{l=0}^{d-1}\left(\frac{d}{r} \tau \frac{\mathrm{d}}{\mathrm{d} \tau}-d \lambda-l\right)\right] I_{\mathbf{k}}^{\mathrm{reg}}(\tau)=0 .
$$

Applying equation (5.8) iteratively to this yields equation (5.5).

To establish equation (5.6), note that $I_{\mathbf{k}}^{\mathrm{reg}}(\tau)$ satisfies

$$
\prod_{j=1}^{N} \prod_{l=0}^{M_{j}-1}\left(-\frac{c_{j}}{r} \tau \frac{\mathrm{d}}{\mathrm{d} \tau}-l-m_{j}(\mathbf{g})\right) I_{\mathbf{k}(\mathbf{g})}^{\mathrm{reg}}(\tau)=I_{\mathbf{k}}^{\mathrm{reg}}(\tau) .
$$

Applying equation (5.8) iteratively to this yields the desired result.

COROLlary 5.7. The function $\mathbb{I}_{\mathbf{k}}\left(u=q^{1 / r}\right)$ satisfies

$$
\left[\prod_{j=1}^{N} \prod_{l=0}^{c_{j}-1}\left(c_{j} q \frac{\mathrm{d}}{\mathrm{d} q}-l-a(\mathbf{k})^{j}\right)-q \prod_{l=0}^{d-1}\left(-d q \frac{\mathrm{d}}{\mathrm{d} q}-d \lambda-l\right)\right] \mathbb{I}_{\mathbf{k}}\left(u=q^{1 / r}\right)=0
$$

for all $g \in \bar{G}$. Furthermore, given $\mathbf{k} \in\left(\mathbb{Z}_{\geqslant 0}\right)^{\bar{G}}$, with the same notation as in the previous lemma, we have

$$
\prod_{j=1}^{N} \prod_{l=0}^{M_{j}-1}\left(c_{j} q \frac{\mathrm{d}}{\mathrm{d} q}-l-m_{j}(\mathbf{g})\right) \mathbb{I}_{\mathbf{k}(\mathbf{g})}\left(u=q^{1 / r}\right)=\mathbb{I}_{\mathbf{k}}\left(u=q^{1 / r}\right) .
$$

Proof. This is a consequence of Lemma 5.6 and the change of variables $q=u^{r}$.

Remark 5.8. Note that, still using the notation of the previous lemma, one can also check that

$$
\prod_{j=1}^{N} \prod_{l=0}^{M_{j}-1}\left(c_{j} q \frac{\mathrm{d}}{\mathrm{d} q}-l-m_{j}(\mathbf{g})\right) I_{\mathbf{k}(\mathbf{g})}^{\mathcal{Y}}(q, z=1)=I_{\mathbf{k}}^{\mathcal{Y}}(q, z=1) .
$$

Corollary 5.9. For all $\mathbf{g} \in \bar{G}$, there exists a unique linear transformation

$$
L_{\mathbf{g}}: H_{\mathbf{g}}^{*}(\mathcal{Y}) \rightarrow H_{\mathbf{g}}^{*}(\mathcal{X})
$$

such that for all $\mathbf{k} \in\left(\mathbb{Z}_{\geqslant 0}\right)^{\bar{G}}$ satisfying $\prod_{g \in \bar{G}} g^{k_{g}}=\mathbf{g}$,

$$
L_{\mathbf{g}} \cdot I_{\mathbf{k}}^{\mathcal{Y}}(q, z=1)=\mathbb{I}_{\mathbf{k}}\left(u=q^{1 / r}\right) .
$$

Proof. Since the components of $I_{\mathbf{k}}^{\mathcal{Y}}(q, z=1)$ are a complete set of solutions to the Picard-Fuchs equation (5.9), by Corollary 5.7 there exists a unique linear transformation $L_{\mathbf{g}}: H_{\mathbf{g}}^{*}(\mathcal{Y}) \rightarrow H_{\mathbf{g}}^{*}(\mathcal{X})$ such that

$$
L_{\mathbf{g}} \cdot I_{\mathbf{k}(\mathbf{g})}^{\mathcal{Y}}(q, z=1)=\mathbb{I}_{\mathbf{k}(\mathbf{g})}\left(u=q^{1 / r}\right),
$$

where $\mathbf{k}(\mathbf{g}) \in\left(\mathbb{Z}_{\geqslant 0}\right)^{\bar{G}}$ is defined as in (5.7). Applying the differential operator

$$
\prod_{j=1}^{N} \prod_{l=0}^{M_{j}-1}\left(c_{j} q \frac{\mathrm{d}}{\mathrm{d} q}-l-m_{j}(\mathbf{g})\right)
$$

from Corollary 5.9 to the equation above, we conclude that (5.12) holds whenever $\prod_{g \in \bar{G}} g^{k_{g}}$ $=\mathrm{g}$. 


\section{P. Acosta And M. Shoemaker}

Proof of Theorem 5.1. By Corollary 5.9, for all $\mathbf{g} \in \bar{G}$, there exists a unique linear transformation $L_{\mathbf{g}}: H_{\mathbf{g}}^{*}(\mathcal{Y}) \longrightarrow H_{\mathbf{g}}^{*}(\mathcal{X})($ recall Notations 4.4 and 4.5$)$ such that $L_{\mathbf{g}} \cdot I_{\mathbf{k}}^{\mathcal{Y}}(q, \boldsymbol{t}, z=1)=\mathbb{I}_{\mathbf{k}}\left(u=q^{1 / r}\right)$ whenever $\prod_{g \in \bar{G}} g^{k_{g}}=\mathrm{g}$. We define $L$ to be the block-diagonal sum

$$
L=\bigoplus_{\mathbf{g} \in \bar{G}} L_{\mathbf{g}}: H_{\mathrm{CR}, T}^{*}(\mathcal{Y})=\bigoplus_{\mathbf{g} \in \bar{G}} H_{\mathbf{g}}^{*}(\mathcal{Y}) \longrightarrow H_{\mathrm{CR}, T}^{*}(\mathcal{X})=\bigoplus_{\mathbf{g} \in \bar{G}} H_{\mathbf{g}}^{*}(\mathcal{X})
$$

It immediately follows that $L \cdot I^{\mathcal{Y}}(q, \boldsymbol{t}, z=1)=\mathbb{I}\left(u=q^{1 / r}\right)$. We now compute its asymptotic expansion:

$$
\begin{aligned}
L \cdot I^{\mathcal{Y}}(q, \boldsymbol{t}, z=1) & =\mathbb{I}\left(u=q^{1 / r}\right)=\sum_{\mathbf{k} \in\left(\mathbb{Z}_{\geqslant 0}\right)^{\bar{G}}} \prod_{g \in \bar{G}} \frac{\left(t^{g}\right)^{k_{g}}}{k_{g} !} \mathbb{I}_{\mathbf{k}}\left(u=q^{1 / r}\right) \\
& \sim \sum_{\mathbf{k} \in\left(\mathbb{Z}_{\geqslant 0}\right)^{\bar{G}}} \prod_{g \in \bar{G}} \frac{\left(t^{g}\right)^{k_{g}}}{k_{g} !} I_{\mathbf{k}}^{\mathcal{X}}\left(t=q^{-1 / d}, z=1\right) \quad \text { as } q \rightarrow \infty \\
& =I^{\mathcal{X}}\left(t=q^{-1 / d}, \boldsymbol{t}, z=1\right) .
\end{aligned}
$$

This concludes the proof of the theorem.

\subsection{The correspondence for $\operatorname{disc}(|f|)<0$}

For the case where $\operatorname{disc}(|f|)<0$, we have a result analogous to Theorem 5.1. In this case, however, the roles of $\mathcal{Y}$ and $\mathcal{X}$ are interchanged. This means that it is possible to obtain the genus zero Gromov-Witten invariants of $\mathcal{Y}$ from the genus zero invariants of $\mathcal{X}$.

Theorem 5.10. Let $\operatorname{disc}(|f|)<0$. There exists a unique linear transformation

$$
L: H_{\mathrm{CR}, T}^{*}(\mathcal{X}) \longrightarrow H_{\mathrm{CR}, T}^{*}(\mathcal{Y})
$$

such that

$$
L \cdot I^{\mathcal{X}}(t, \boldsymbol{t}, z=1) \sim I^{\mathcal{Y}}\left(q=t^{-d}, \boldsymbol{t}, z=1\right) \quad \text { as } t \rightarrow \infty
$$

The proof of this result is almost identical to the proof of Theorem 5.1 once we interchange the roles of $\mathcal{X}$ and $\mathcal{Y}$.

As in the case $\operatorname{disc}(|f|)>0$, we have the following important consequence.

Corollary 5.11. If $\operatorname{disc}(|f|)<0$, then the genus zero Gromov-Witten invariants of $\mathcal{Y}$ are completely determined by the genus zero Gromov-Witten invariants of $\mathcal{X}$.

\section{FJRW theory and Landau-Ginzburg correspondences}

In this section, we prove that the FJRW theory of the pair $(W, G)$ from Section 4 is related to the Gromov-Witten theory of the hypersurface $\mathcal{Z}:=\{W=0\} \subset \mathbb{P}(G)$ via asymptotic expansion as in Section 5. This generalizes the results of the first author in [Aco14] beyond the case $\mathbb{P}(G)=\mathbb{P}\left(c_{1}, \ldots, c_{N}\right)$ and may be viewed as extending the Landau-Ginzburg/Calabi-Yau correspondence [CR10, CIR14, PS16, LPS16] to the setting where $\mathcal{Z}$ is no longer Calabi-Yau. For simplicity, we will term these types of theorems Landau-Ginzburg correspondences. While they are not strictly a consequence of Theorems 5.1 and 5.10 , we will show how the proofs above may be modified slightly to yield the desired correspondences. 


\section{QuANTUM COHOMOLOGY OF BLOWUPS}

\subsection{Setup}

We first recall notation from Section 4 . We are given a Fermat polynomial $W=X_{1}^{d / c_{1}}+\cdots+X_{N}^{d / c_{N}}$ with $\operatorname{gcd}\left(c_{1}, \ldots, c_{N}\right)=1$ and a group $G$ of diagonal automorphisms of $W$. The polynomial $W$ is quasi-homogeneous of degree $d$ :

$$
W\left(\alpha^{c_{1}} X_{1}, \ldots, \alpha^{c_{N}} X_{N}\right)=\alpha^{d} W\left(X_{1}, \ldots, X_{N}\right)
$$

for all $\alpha \in \mathbb{C}$. We assume that $G$ contains the distinguished element $\mathfrak{j}=\exp \left(2 \pi i \operatorname{diag}\left(q_{1}, \ldots, q_{N}\right)\right)$, where $q_{j}=c_{j} / N$. For the correspondence to hold, we also require that the line bundle $\mathscr{O}(-d) \rightarrow$ $\mathbb{P}(G)$ be pulled back from the coarse underlying space of $\mathbb{P}(G)$. This corresponds to the somewhat technical condition that all $g \in G$ which fix at least one coordinate lie in $\mathrm{SL}_{N}(\mathbb{C})$. We call $(W, G)$ a Landau-Ginzburg pair.

\subsection{FJRW theory}

Given a pair $(W, G)$ as above, one can apply the general theory of [FJR13] to construct FJRW invariants of $(W, G)$. Similarly to the case of Gromov-Witten theory, these invariants are defined as integrals of a virtual class on certain moduli spaces lying over $\overline{\mathscr{M}}_{g, n}$. As such, FJRW theory enjoys many of the same properties as Gromov-Witten theory.

In general, the definition of FJRW invariants is quite involved, but in genus zero, when $W$ is a Fermat polynomial, the situation simplifies greatly. In what follows, we will always assume that the conditions on $(W, G)$ given above are satisfied.

We first define the state space.

DeFinition 6.1. Given a Landau-Ginzburg pair $(W, G)$, the narrow FJRW state space is given by

$$
\mathcal{H}_{\mathrm{FJRW}}^{\mathrm{nar}}(W, G):=\bigoplus_{g \in G_{\mathrm{nar}}} \mathbb{C} \phi_{g},
$$

where

$$
G_{\text {nar }}:=\left\{g \in G \mid g \text { j fixes only the origin in } \mathbb{C}^{N}\right\}
$$

and $\phi_{g}$ is a vector formally associated with $g \in G_{\text {nar }}$.

Definition 6.2. Given a Landau-Ginzburg pair $(W, G)$ as above, the moduli space $W_{h, n ; G}$ of $W$ structures parametrizes families of orbifold curves $\mathcal{C}$ together with an $N$-tuple $\left(\mathcal{L}_{j}, \varphi_{j}\right)$ such that

$-\mathcal{C}$ has $\mu_{d}$ isotropy at each marked point and node, and the coarse space $|\mathcal{C}|$ is a (family of) $n$-pointed genus $h$ stable curve;

- $\mathcal{L}_{j}$ is a line bundle on $\mathcal{C}$;

- $\varphi_{j}$ is an isomorphism $\varphi_{j}: \mathcal{L}_{j}^{\otimes d} \stackrel{\sim}{\rightarrow} \omega_{\mathcal{C}, \log }^{\otimes c_{j}}$; and

- for any Laurent monomial $\prod_{j=1}^{N} X_{j}^{b_{j}}$ invariant under $G$,

$$
\bigotimes_{j=1}^{N} \mathcal{L}_{j}^{\otimes b_{j}} \cong \omega_{\mathcal{C}, \log } .
$$

See [FJR13, LPS16] for more details.

As a consequence of the condition in the third item above, given a point $\left(\mathcal{C}, \mathcal{L}_{j}, \phi_{j}\right)$ in $W_{h, n ; G}$, at each marked point $p_{i}$, the isotropy acts on fibers of $\oplus_{j=1}^{N} \mathcal{L}_{j}$ by an element of $G$. One may therefore decompose $W_{h, n, G}$ into a union of open and closed substacks based on the action of the 


\section{P. Acosta And M. Shoemaker}

corresponding isotropy group. Let

$$
W_{h, n, G}\left(g_{1}, \ldots, g_{n}\right)
$$

denote the substack where the isotropy generator at $p_{i}$ acts by $g_{i}$.

In analogy to Gromov-Witten theory, FJRW invariants are defined by integrating against a virtual cycle on $W_{h, n ; G}$. When $W$ is a Fermat polynomial and the genus is zero, one can prove [FJR13] that

$$
R^{0} \pi_{*}\left(\oplus_{j=1}^{N} \mathcal{L}_{j}\right)=0
$$

and

$$
-R \pi_{*}\left(\oplus_{j=1}^{N} \mathcal{L}_{j}\right)=R^{1}\left(\oplus_{j=1}^{N} \mathcal{L}_{j}\right)[-1]
$$

is a vector bundle. Let $\phi_{g_{1}}, \ldots, \phi_{g_{n}}$ be elements of the state space $\mathcal{H}_{\mathrm{FJRW}}^{\text {nar }}(W, G)$, and let $a_{1}, \ldots, a_{n}$ be non-negative integers. By axiom (5a) of [FJR13, Theorem 4.1.8], one defines

$$
\left\langle\psi^{a_{1}} \phi_{g_{1}}, \ldots, \psi^{a_{n}} \phi_{g_{n}}\right\rangle_{0, n}^{(W, G)}:=\bar{d}^{N} \int_{W_{0, n, G}\left(g_{1} j, \ldots, g_{n} \mathrm{j}\right)} \frac{\prod_{i=1}^{n} \psi_{i}^{a_{i}}}{e\left(R \pi_{*}\left(\oplus_{j=1}^{N} \mathcal{L}_{j}\right)^{\vee}\right)} .
$$

FJRW theory is a cohomological field theory. Furthermore, all of the axioms of an axiomatic Gromov-Witten theory [Lee09, LPS16] are satisfied. Therefore, quantum cohomology, the Dubrovin connection, the $J$-function, and $I$-functions may all be defined and behave in the same manner as in Gromov-Witten theory. Thus, Section 3 transfers almost word for word to the FJRW setting. See [LPS16, Section 3] for more details. We will denote the $J$-function and $I$-functions coming from the FJRW theory of $(W, G)$ by $J^{(W, G)}$ and $I^{(W, G)}$, respectively.

Remark 6.3. The reader may observe a strange shift in equation (6.1), the $\phi_{g_{i}}$ insertion at the $i$ th marked point corresponds to an isotropy action of $g_{i}$ j. This shift is chosen such that the $\phi_{\mathrm{id}}$ element of the state space is the identity in quantum cohomology.

Remark 6.4. FJRW theory is defined for a more general class of insertions corresponding to $g \in G \backslash G_{\text {nar }}$ (in analogy with primitive cohomology classes of a hypersurface). We will content ourselves in this paper with statements relating the narrow invariants from $G_{\text {nar }}$ to the ambient cohomology of $\mathcal{Z}$.

\subsection{I-functions}

Rather than directly compute the relevant $I$-functions for the Landau-Ginzburg correspondences, we will apply two results, the multiple log-canonical (MLK) and quantum Serre duality (QSD) correspondences to obtain $I^{(W, G)}$ and $I^{\mathcal{Z}}$ from $I^{\mathcal{X}}$ and $I^{\mathcal{Y}}$, respectively. The MLK correspondence says, roughly, that $I^{(W, G)}$ is related to $I^{\mathcal{X}}$ by differentiation, linear transformation, and a nonequivariant limit. As a consequence, we will see that the Picard-Fuchs differential equations satisfied by $I^{(W, G)}$ are closely related to those for $I^{\mathcal{X}}$ described in the previous section. The QSD correspondence is analogous.

6.3.1 The I-function of $\mathcal{X}$. Applying the MLK correspondence [LPS16, Theorem 5.12], an FJRW $I$-function for $(W, G)$ is given by

$$
I^{(W, G)}(t, \boldsymbol{t}, z)=\lim _{\lambda \mapsto 0} \Delta^{\circ}\left(z t \frac{\mathrm{d}}{\mathrm{d} t} I^{\mathcal{X}}(t, \boldsymbol{t}, z)\right),
$$




\section{QUANTUM COHOMOLOGY OF BLOWUPS}

where $\Delta^{\circ}$ is the linear map defined by

$$
\Delta^{\circ}:=\mathbb{1}_{g} \mapsto \begin{cases}(-1)^{\mathrm{age}(g)} \phi_{g \mathrm{j}^{-1}} & \text { if } g \text { fixes only the origin } \\ 0 & \text { otherwise }\end{cases}
$$

We obtain

$$
\begin{aligned}
I^{(W, G)}(t, \boldsymbol{t}, z)= & z \sum_{\mathbf{k} \in\left(\mathbb{Z}_{\geqslant 0}\right)^{\bar{G}}} \prod_{g \in \bar{G}} \frac{\left(t^{g}\right)^{k_{g}} z^{(\operatorname{age}(g)-1) k_{g}}}{k_{g} !} \sum_{k_{0} \geqslant 0} \frac{t^{k_{0}+1} z^{1+\left(k_{0}+1\right) r / d}}{z^{\sum_{j}\left\langle\left(k_{0}+1\right) q_{j}+a(\mathbf{k})^{j}\right\rangle} k_{0} !} \\
& \times \prod_{j=1}^{N} \frac{\Gamma\left(1-\left\langle\left(k_{0}+1\right) q_{j}+a(\mathbf{k})^{j}\right\rangle\right)}{\Gamma\left(1-\left(k_{0}+1\right) q_{j}-a(\mathbf{k})^{j}\right)}(-1)^{\operatorname{age}\left(\mathrm{j}^{k_{0}} \prod_{g} g^{\left.k_{g}\right)}\right.} \phi_{\mathbf{j}^{k_{0}}} \prod_{g} g^{k_{g}},
\end{aligned}
$$

where $\phi_{g}$ is understood to be zero if $g \notin G_{\text {nar }}$.

Now, given $\mathbf{g} \in \bar{G}$, consider the $t^{\mathbf{g}}$-coefficient of $I^{(W, G)}(t, \boldsymbol{t}, z)$, and denote it by $I_{\mathbf{g}}^{(W, G)}(t, \boldsymbol{t}, z)$ :

$$
\begin{aligned}
I_{\mathbf{g}}^{(W, G)}(t, \boldsymbol{t}, z)= & z \sum_{k_{0} \geqslant 0} \frac{t^{k_{0}+1} z^{1+\left(k_{0}+1\right) r / d}}{z^{\sum_{j}\left\langle\left(k_{0}+1\right) q_{j}+m_{j}(\mathbf{g})\right\rangle} k_{0} !} \\
& \times \prod_{j=1}^{N} \frac{\Gamma\left(1-\left\langle\left(k_{0}+1\right) q_{j}+m_{j}(\mathbf{g})\right\rangle\right)}{\Gamma\left(1-\left(k_{0}+1\right) q_{j}-m_{j}(\mathbf{g})\right)}(-1)^{\operatorname{age}\left(\mathrm{j}^{k_{0}} \prod_{g} g^{k g}\right)} \phi_{\mathrm{j} k_{0}} \mathbf{g} .
\end{aligned}
$$

Note that since $\phi_{j^{k} \mathbf{g}}=0$ if $j^{k} \mathbf{g}$ is not narrow, the function $I_{\mathbf{g}}^{(W, G)}(t, \boldsymbol{t}, z)$ is supported on the space

$$
\mathcal{H}_{\mathrm{g}}^{\text {nar }}(W, G):=\bigoplus_{\substack{0 \leqslant k \leqslant d-1 \\ j^{k} \mathbf{g} \in G_{\text {nar }}}} \mathbb{C} \phi_{j^{k} g}
$$

of dimension $\operatorname{dim}\left(H_{\mathbf{g}}^{*}(\mathcal{X})\right)-\#\left\{0 \leqslant k \leqslant d-1 \mid j^{k} \mathbf{g}\right.$ fixes a coordinate $\}$.

We claim that $I_{\mathrm{g}}^{(W, G)}(t, \boldsymbol{t}, z)$ satisfies a Picard-Fuchs equation obtained from that of $I_{\mathbf{g}}^{\mathcal{X}}(t, \boldsymbol{t}, z)$ by removing factors. The non-equivariant limit of the original equation for $\mathcal{X}$ is

$$
\mathcal{D}_{t, \mathbf{g}}((f(t, z))=0,
$$

where

$$
\mathcal{D}_{t, \mathbf{g}}=\prod_{l=0}^{d-1}\left(z t \frac{\mathrm{d}}{\mathrm{d} t}-l z\right)-t^{d} \prod_{j=1}^{N} \prod_{l=0}^{c_{j}-1}\left(\left(-c_{j} / d\right) z t \frac{\mathrm{d}}{\mathrm{d} t}-l z-m_{j}(\mathbf{g}) z\right) .
$$

First, note that by construction of our splitting $\bar{G}$, we have $m_{1}(\mathbf{g})=0$. Thus, we can factor a $z t \frac{\mathrm{d}}{\mathrm{d} t}$ from the second term of $\mathcal{D}_{t, \mathbf{g}}$ since the $j=1, l=0$ factor in the second product is a multiple of $z t \frac{\mathrm{d}}{\mathrm{d} t}$. It is immediate that $I_{\mathrm{g}}^{(W, G)}(t, t, z)$ is annihilated by this reduced operator, because it was obtained by applying $z t \frac{\mathrm{d}}{\mathrm{d} t}$ to $I_{\mathbf{g}}^{\mathcal{Z}}(t, \boldsymbol{t}, z)$. Next, assume that $\mathfrak{j}^{k} \mathbf{g}$ fixes coordinate $i$ for some $1 \leqslant k \leqslant d-1$. We claim that $\mathcal{D}_{t, \mathbf{g}}$ contains a factor of $\left(z t \frac{\mathrm{d}}{\mathrm{d} t}-k z\right)$ in the first product.

First, note that $m_{i}(\mathbf{g})=f c_{i} / d$ for some $1 \leqslant f \leqslant d / c_{i}-1$; thus $k=m d / c_{i}-f$ for $1 \leqslant m \leqslant c_{i}$. Consider the factor $\left(\left(-c_{j} / d\right) z t \frac{\mathrm{d}}{\mathrm{d} t}-l z-m_{j}(\mathbf{g}) z\right)$ in the second product in $\mathcal{D}_{t, \mathbf{g}}$ for $l=c_{i}-m$. 


\section{P. Acosta And M. Shoemaker}

Commuting this with $t^{d}$ yields

$$
\begin{aligned}
t^{d}\left(\left(-c_{i} / d\right) z t \frac{\mathrm{d}}{\mathrm{d} t}-\left(c_{i}-m\right) z-m_{i}(\mathbf{g}) z\right) & =\left(\left(-c_{i} / d\right)\left(z t \frac{\mathrm{d}}{\mathrm{d} t}-d\right)-\left(c_{i}-m\right) z-m_{i}(\mathbf{g}) z\right) t^{d} \\
& =-\left(c_{i} / d\right)\left(z t \frac{\mathrm{d}}{\mathrm{d} t}-\left(m d / c_{i}-m_{i}(\mathbf{g}) d / c_{i}\right)\right) t^{d} \\
& =-\left(c_{i} / d\right)\left(z t \frac{\mathrm{d}}{\mathrm{d} t}-k\right) t^{d} .
\end{aligned}
$$

We can thus factor $\left(z t \frac{\mathrm{d}}{\mathrm{d} t}-k\right)$ from the first product in $\mathcal{D}_{t, \mathbf{g}}$ for every power of $k$ such that $\mathfrak{j}^{k} \mathbf{g}$ fixes a coordinate. We obtain

$$
\mathcal{D}_{t, \mathbf{g}}=\prod_{\left\{\begin{array}{c}
1 \leqslant k \leqslant d-1 \\
j^{k} \mathbf{g} \text { fixes a coordinate }
\end{array}\right.}\left(z t \frac{\mathrm{d}}{\mathrm{d} t}-k\right) \cdot \mathcal{D}_{t, \mathbf{g}}^{\mathrm{irr}} \cdot z t \frac{\mathrm{d}}{\mathrm{d} t} .
$$

One can easily check that the $I$-function (6.3) satisfies the Picard-Fuchs equation $\mathcal{D}_{t, \mathbf{g}}^{\mathrm{irr}}((f(t, z))$ $=0$.

6.3.2 The $I$-function of $\mathcal{Z}$. Let $i: \mathcal{Z} \rightarrow \mathbb{P}(G)$ be the inclusion of the hypersurface defined by the equation $\{W=0\}$. By quantum Serre duality of Coates-Givental [CG07] and Tseng [Tse10], an $I$-function for $\mathcal{Z}$ is given by

$$
I^{\mathcal{Z}}(q, \boldsymbol{t}, z)=\lim _{\lambda \mapsto 0} i^{*}\left(\frac{z q \frac{\mathrm{d}}{\mathrm{d} q} I^{\mathcal{Y}}( \pm q, \boldsymbol{t}, z)}{-d(\lambda+H)}\right),
$$

where $\pm q$ denotes the substitution $q^{k_{0} / d} \mapsto(-1)^{k_{0}} q^{k_{0} / d}$. We obtain

$$
\begin{aligned}
I^{\mathcal{Z}}(q, \boldsymbol{t}, z)= & z q^{H / z} \sum_{\mathbf{k} \in\left(\mathbb{Z}_{\geqslant 0}\right)^{\bar{G}}} \prod_{g \in \bar{G}} \frac{\left(t^{g}\right)^{k_{g}} z^{(\operatorname{age}(g)-1) k_{g}}}{k_{g} !} \sum_{k_{0} \geqslant 0} \frac{(-1)^{k_{0}} q^{k_{0} / d}}{z^{k_{0} r / d+\sum_{j}\left\langle k_{0} q_{j}-a(\mathbf{k})^{j}\right\rangle}} \\
& \times \frac{\Gamma(-d H / z)}{\Gamma\left(-k_{0}-d H / z\right)} \prod_{j=1}^{N} \frac{\Gamma\left(1+c_{j} H / z-\left\langle-k_{0} q_{j}+a(\mathbf{k})^{j}\right\rangle\right)}{\Gamma\left(1+c_{j} H / z+k_{0} q_{j}-a(\mathbf{k})^{j}\right)} \tilde{\mathbb{1}}_{j^{-k_{0}} \prod_{g} g^{k_{g}}},
\end{aligned}
$$

where we now consider cohomology classes $H^{k} \tilde{\mathbb{1}}_{\mathfrak{j}^{-k_{0}}} \prod_{g} g^{k_{g}}$ as elements of $H_{\mathrm{CR}}^{*}(\mathcal{Z})$ via the pullback to the hypersurface. The function $I^{\mathcal{Z}}(q, \boldsymbol{t}, z)$ gives a big $I$-function for the ambient part of the Gromov-Witten theory of $\mathcal{Z}$. In other words, $I^{\mathcal{Z}}(q, \boldsymbol{t}, z)$ determines the quantum cohomology ring of $\mathcal{Z}$ restricted to classes in $H_{\mathrm{CR}}^{\text {amb }}(\mathcal{Z}) \subset H_{\mathrm{CR}}^{*}(\mathcal{Z})$ defined as the image of $i^{*}: H_{\mathrm{CR}}^{*}(\mathbb{P}(G)) \rightarrow$ $H_{\mathrm{CR}}^{*}(\mathcal{Z})$.

As in the previous case, given $\mathbf{g} \in \bar{G}$, let $I_{\mathbf{g}}^{\mathcal{Z}}(t, \boldsymbol{t}, z)$ be the part of $I^{\mathcal{Z}}(t, \boldsymbol{t}, z)$ coming from the $t^{\mathrm{g}}$-coefficient:

$$
\begin{aligned}
I_{\mathbf{g}}^{\mathcal{Z}}(q, \boldsymbol{t}, z)= & z q^{H / z} \sum_{k_{0} \geqslant 0} \frac{(-1)^{k_{0}} q^{k_{0} / d}}{z^{k_{0} r / d+\sum_{j}\left\langle k_{0} q_{j}-m_{j}(\mathbf{g})\right\rangle}} \\
& \times \frac{\Gamma(-d H / z)}{\Gamma\left(-k_{0}-d H / z\right)} \prod_{j=1}^{N} \frac{\Gamma\left(1+c_{j} H / z-\left\langle-k_{0} q_{j}+a(\mathbf{k})^{j}\right\rangle\right)}{\Gamma\left(1+c_{j} H / z+k_{0} q_{j}-m_{j}(\mathbf{g})\right)} \tilde{\mathbb{1}}_{\mathfrak{j}^{-k_{0}}} \prod_{g} g^{k_{g}} .
\end{aligned}
$$

Note that top powers of $H$ on any connected component of $I \mathbb{P}(G)$ will vanish under the pullback. 


\section{QUANTUM COHOMOLOGY OF BLOWUPS}

Thus, $I_{\mathbf{g}}^{\mathcal{Z}}(t, \boldsymbol{t}, z)$ is supported on the space

$$
H_{\mathbf{g}}^{*}(\mathcal{Z}):=i^{*}\left(H^{*}\left(\cup_{0 \leqslant k \leqslant d-1} \mathbb{P}(G)_{\mathbf{j}} \mathbf{g}\right)\right)
$$

of $\operatorname{dimension} \operatorname{dim}\left(H_{\mathbf{g}}^{*}(\mathcal{Y})\right)-\#\left\{0 \leqslant k \leqslant d-1 \mid j^{k} \mathbf{g}\right.$ fixes a coordinate $\}$.

\subsection{Landau-Ginzburg correspondences}

We begin this section by establishing the Landau-Ginzburg/Fano correspondence. We assume $\sum c_{j}>d$, which implies that $\mathcal{Z}$ is a Fano hypersurface. The main result we prove in this section is that the genus zero Gromov-Witten theory of $\mathcal{Z}$ completely determines the genus zero FJRW theory of the pair $(W, G)$. The technical details of the proof of this result are almost identical to the details found Section 5. For this reason, we avoid some computations.

We start by noting that $I_{\mathbf{g}}^{\mathcal{Z}}(q, z)$ satisfies the equation $\mathcal{D}_{q, \mathbf{g}}^{\text {irr }}\left(I_{\mathbf{g}}^{\mathcal{Z}}(q, z)\right)=0$, where the differential operator $\mathcal{D}_{q, \mathbf{g}}^{\mathrm{irr}}$ is equal to $\mathcal{D}_{t, \mathbf{g}}^{\mathrm{irr}}$ of $(6.4)$, but with the change of variables $q=(-t)^{d}$.

Since the degree of the differential operator $\mathcal{D}_{q, \mathbf{g}}^{\text {irr }}$ is the same as the dimension of $H_{\mathbf{g}}^{*}(\mathcal{Z})$, it follows that the components of $I_{\mathbf{g}}^{\mathcal{Z}}(q, z)$ are a complete set of solutions to the equation $\mathcal{D}_{q, \mathbf{g}}^{\text {irr }}((f(q, z))=0$. Next, we construct a holomorphic function whose asymptotic expansion at infinity is given by $I_{\mathrm{g}}^{(W, G)}$. Define the regularized version of $I_{\mathrm{g}}^{(W, G)}$ by

$$
\begin{aligned}
I_{\mathbf{g}}^{(W, G), \mathrm{reg}}(\tau):= & \sum_{k_{0} \geqslant 0} \frac{(-1)^{k_{0}+1} \tau^{r\left(k_{0}+1\right) / d}}{\Gamma\left(1+\frac{r}{d}\left(k_{0}+1\right)\right) k_{0} !} \\
& \times \prod_{j=1}^{N} \frac{\Gamma\left(1-\left\langle\left(k_{0}+1\right) q_{j}+m_{j}(\mathbf{g})\right\rangle\right)}{\Gamma\left(1-\left(k_{0}+1\right) q_{j}-m_{j}(\mathbf{g})\right)}(-1)^{\left.\operatorname{age}^{\left(j^{k}\right.} \prod_{g} g^{k_{g}}\right)} \phi_{\mathbf{j}} k_{0} \mathbf{g} .
\end{aligned}
$$

This series converges on a disk of finite radius centered at $\tau=0$. Moreover, it satisfies a regularized Picard-Fuchs equation $\mathcal{D}_{\tau, \mathbf{g}}^{\text {irr,reg }}\left(I_{\mathbf{g}}^{(W, G), \text { reg }}(\tau)\right)=0$ with regular singularities at $\tau=0, \infty$ and at $\tau$ satisfying $(\tau / r)^{r}=d^{d} \prod_{j=1}^{N} c_{j}^{-c_{j}}$. Thus, $I_{\mathrm{g}}^{(W, G), \text { reg }}(\tau)$ can be analytically continued to $\tau=\infty$ along any ray that avoids these singularities. Define

$$
\mathbb{I}_{\mathbf{g}}^{(W, G)}(u):=u \int_{0}^{\infty} e^{-u \tau} I_{\mathbf{g}}^{(W, G), \mathrm{reg}}(\tau) \mathrm{d} \tau,
$$

where the ray on integration avoids the singular points of $\mathcal{D}_{\tau, \mathbf{g}}^{\text {irreg }}$. By Watson's lemma, we have

$$
\mathbb{I}_{\mathbf{g}}^{(W, G)}\left(u=q^{1 / r}\right) \sim I_{\mathrm{g}}^{(W, G)}\left(t=-q^{-1 / d}, z=1\right) \quad \text { as } q \rightarrow \infty .
$$

After a computation similar to the one found in the proof of Corollary 5.7, one sees that $\mathcal{D}_{q, \mathbf{g}}^{\mathrm{irr}} \cdot \mathbb{I}_{\mathbf{g}}^{(W, G)}\left(u=q^{1 / r}\right)=0$. We now prove a result similar to Corollary 5.9.

Lemma 6.5. For each $\mathrm{g} \in \bar{G}$, there exists a unique linear transformation

$$
L_{\mathbf{g}}: H_{\mathbf{g}}^{*}(\mathcal{Z}) \rightarrow \mathcal{H}_{\mathrm{FJRW}}^{\text {nar }}(W, G)
$$

such that for all $\mathbf{k} \in\left(\mathbb{Z}_{\geqslant 0}\right)^{\bar{G}}$ satisfying $\prod_{g} g^{k_{g}}=\mathbf{g}$,

$$
L_{\mathbf{g}} \cdot I_{\mathbf{k}}^{\mathcal{Z}}(q, z=1)=\mathbb{I}_{\mathbf{k}}^{(W, G)}\left(u=q^{1 / r}\right) .
$$

Proof. Since $\mathcal{D}_{q, \mathbf{g}}^{\mathrm{irr}} \cdot \mathbb{I}_{\mathbf{g}}^{(W, G)}\left(u=q^{1 / r}\right)=0$ and the components of $I_{\mathbf{g}}^{\mathcal{Z}}(q, z=1)$ are a complete set of solutions to $\mathcal{D}_{q, \mathbf{g}}^{\text {irr }}\left((f(q, z))=0\right.$, there exists a unique linear transformation $L_{\mathbf{g}}: H_{\mathbf{g}}^{*}(\mathcal{Z}) \rightarrow$ $\mathcal{H}_{\mathrm{g}}^{\text {nar }}(W, G)$ such that

$$
L_{\mathbf{g}} \cdot I_{\mathbf{g}}^{\mathcal{Z}}(q, z=1)=\mathbb{I}_{\mathbf{g}}^{(W, G)}\left(u=q^{1 / r}\right)
$$




\section{P. Acosta And M. Shoemaker}

The proof of the second part of the lemma follows an argument identical to the one found in Corollary 5.9; note that equations (5.10) and (5.11) still hold in this setting.

Corollary 6.6. Given $\mathbf{g} \in \bar{G}$, there exists a unique linear transformation $L_{\mathbf{g}}: H_{\mathbf{g}}^{*}(\mathcal{Z}) \rightarrow$ $\mathcal{H}_{\mathrm{g}}^{\text {nar }}(W, G)$ such that

$$
L_{\mathbf{g}} \cdot I_{\mathbf{g}}^{\mathcal{Z}}(q, z=1) \sim I_{\mathbf{g}}^{(W, G)}\left(t=-q^{-1 / d}, z=1\right) \quad \text { as } q \rightarrow \infty .
$$

Proof. This result easily follows from equation (6.8) and Lemma 6.5.

As a consequence of Lemma 6.5, we obtain the following result.

Theorem 6.7. Let $\sum_{j=1}^{N} c_{j}-d>0$. Then, there exists a unique linear transformation

$$
L^{\mathrm{GW}}: H_{\mathrm{CR}}^{\mathrm{amb}}(\mathcal{Z}) \longrightarrow \mathcal{H}_{\mathrm{FJRW}}^{\mathrm{nar}}(W, G)
$$

such that

$$
L^{\mathrm{GW}} \cdot I^{\mathcal{Z}}(q, \boldsymbol{t}, z=1) \sim I^{(W, G)}\left(t=-q^{-1 / d}, \boldsymbol{t}, z=1\right) \quad \text { as } q \rightarrow \infty .
$$

Proof. Define $L^{\mathrm{GW}}$ to be the block-diagonal transformation given by $L^{\mathrm{GW}}:=\bigoplus_{g \in \bar{G}} L_{\mathbf{g}}$. Then the proof is identical to the proof of Theorem 5.1, but we use Lemma 6.5 and Corollary 6.6 instead of Corollary 5.9.

Remark 6.8. One can uniquely recover $I^{\mathcal{Z}}(q, \boldsymbol{t}, z)$ and $I^{(W, G)}(t, \boldsymbol{t}, z)$ from $I^{\mathcal{Z}}(q, \boldsymbol{t}, 1)$ and $I^{(W, G)}(t, \boldsymbol{t}, 1)$ by using a procedure similar to the one described in Section 5.2.

The following result is a natural consequence of Theorem 6.7.

Corollary 6.9. If $\sum_{j=1}^{N} c_{j}-d>0$, the genus zero FJRW theory of the pair $(W, G)$ is completely determined by the genus zero Gromov-Witten invariants of $\mathcal{Z}$. It follows that the FJRW quantum cohomology ring of $(W, G)$ is determined by the quantum cohomology of $\mathcal{Z}$.

Proof. The genus zero Gromov-Witten invariants of $\mathcal{Z}$ completely determine the Givental $I$ function $I^{\mathcal{Z}}(q, \boldsymbol{t}, z)$. As a consequence of Theorem 6.7, the FJRW $I$-function of the pair $(W, G)$ is uniquely determined by $L^{\mathrm{GW}}$ and power series asymptotic expansion at infinity. By means of Birkhoff factorization, the FJRW $I$-function completely determines the FJRW $J$-function and therefore, the genus zero FJRW theory.

We finish this section by stating a Landau-Ginzburg/general type correspondence. This can be proved in an analogous manner to the Fano case. For the remainder of the section, assume $\sum_{j=1}^{N} c_{j}-d<0$. This condition implies that $\mathcal{Z}$ is a hypersurface of general type. We then have the following results.

ThEOREM 6.10. If $\sum_{j=1}^{N} c_{j}-d<0$, there exists a unique linear transformation

$$
L^{\mathrm{FJRW}}: \mathcal{H}_{\mathrm{FJRW}}^{\mathrm{nar}}(W, G) \longrightarrow H_{\mathrm{CR}}^{\mathrm{amb}}(\mathcal{Z})
$$

such that

$$
L^{\mathrm{FJRW}} \cdot I^{(W, G)}(t, \boldsymbol{t}, z=1) \sim I^{\mathcal{Z}}\left(q=t^{-d}, \boldsymbol{t}, z=1\right) \quad \text { as } t \rightarrow \infty .
$$

Corollary 6.11. If $\sum_{j=1}^{N} c_{j}-d<0$, the genus zero $G W$ theory of $\mathcal{Z}$ is completely determined by the genus zero FJRW theory of the pair $(W, G)$. It follows that the quantum cohomology of $\mathcal{Z}$ is determined by the FJRW quantum cohomology ring of the pair $(W, G)$.

The proofs of these statements are identical to the proofs of Theorems 6.7 and 6.9 after exchanging the roles of $I^{\mathcal{Z}}(q, \boldsymbol{t}, z)$ and $I^{(W, G)}(t, \boldsymbol{t}, z)$. 


\section{QUANTUM COHOMOLOGY OF BLOWUPS}

\section{ACKNOWLEDGEMEnTS}

The authors would like to thank H. Iritani for many useful conversations and for his talks on the crepant transformation conjecture. They are also grateful to their advisor, Y. Ruan, for teaching them much of what they know about Gromov-Witten and FJRW theory. P.A. would like to thank D. Ross for his interest in this work and for fruitful conversations. He also acknowledges the Algebraic Geometry group at the University of Utah for their hospitality during his visit in February of 2015. M.S. would like to thank Y.-P. Lee for initially suggesting the MLK correspondence, on which the present paper relies.

\section{REFERENCES}

Aco14 P. Acosta, Asymptotic expansion and the LG/(Fano, general type) correspondence, 2014, arXiv:1411.4162.

Ado94 A. Adolphson, Hypergeometric functions and rings generated by monomials, Duke Math. J. 73 (1994), no. 2, 269-290; https://doi.org/10.1215/S0012-7094-94-07313-4.

AGV08 D. Abramovich, T. Graber, and A. Vistoli, Gromov-Witten theory of Deligne-Mumford stacks, Amer. J. Math. 130 (2008), no. 5, 1337-1398; https://doi.org/10.1353/ajm.0.0017.

AS16 P. Acosta and M. Shoemaker, Gromov-Witten theory of toric birational transformations, 2016, arXiv:1604.03491.

BCR13 A. Brini, R. Cavalieri, and D. Ross, Crepant resolutions and open strings, 2013, arXiv:1309.4438.

BG09 J. Bryan and T. Graber, The crepant resolution conjecture, Algebraic Geometry (Seattle 2005), Part 1, Proc. Sympos. Pure Math., vol. 80 (Amer. Math. Soc., Providence, RI, 2009), 23-42; https://doi.org/10.1090/pspum/080.1/2483931.

CCIT09 T. Coates, A. Corti, H. Iritani, and H.-H. Tseng, Computing genus-zero twisted GromovWitten invariants, Duke Math. J. 147 (2009), no. 3, 377-438; https://doi.org/10.1215/ 00127094-2009-015.

CCIT15 _ A mirror theorem for toric stacks, Compos. Math. 151 (2015), no. 10, 1878-1912; https://doi.org/10.1112/S0010437X15007356.

CG07 T. Coates and A. Givental, Quantum Riemann-Roch, Lefschetz and Serre, Ann. of Math. 165 (2007), no. 1, 15-53; https://doi.org/10.4007/annals.2007.165.15.

CI14 T. Coates and H. Iritani, A Fock sheaf for Givental quantization, 2014, arXiv:1411.7039.

CIJ14 T. Coates, H. Iritani, and Y. Jiang, The Crepant transformation conjecture for toric complete intersections, 2014, arXiv:1410.0024.

CIR14 A. Chiodo, H. Iritani, and Y. Ruan, Landau-Ginzburg/Calabi-Yau correspondence, global mirror symmetry and Orlov equivalence, Publ. Math. Inst. Hautes Études Sci. 119 (2014), 127-216; https://doi.org/10.1007/s10240-013-0056-z.

CIT09 T. Coates, H. Iritani, and H.-H. Tseng, Wall-crossings in toric Gromov-Witten theory. I. Crepant examples, Geom. Topol. 13 (2009), no. 5, 2675-2744; https://doi.org/10.2140/ gt.2009.13.2675.

CK99 D. A. Cox and S. Katz, Mirror symmetry and algebraic geometry, Math. Surveys Monogr., vol. 68 (Amer. Math. Soc., Providence, RI, 1999; https://doi.org/10.1090/surv/068.

CR02 W. Chen and Y. Ruan, Orbifold Gromov-Witten theory, Orbifolds in Mathematics and Physics (Madison, WI, 2001), Contemp. Math., vol. 310, (Amer. Math. Soc., Providence, RI, 2002), 25-85; https://doi.org/10.1090/conm/310/05398.

CR04 _ A new cohomology theory of orbifold, Comm. Math. Phys. 248 (2004), no. 1, 1-31; https://doi.org/10.1007/s00220-004-1089-4. 


\section{P. Acosta And M. Shoemaker}

CR10 A. Chiodo and Y. Ruan, Landau-Ginzburg/Calabi-Yau correspondence for quintic three-folds via symplectic transformations, Invent. Math. 182 (2010), no. 1, 117-165; https://doi.org/ 10.1007/s00222-010-0260-0.

CR13 T. Coates and Y. Ruan, Quantum cohomology and crepant resolutions: a conjecture, Ann. Inst. Fourier (Grenoble) 63 (2013), no. 2, 431-478; https://doi.org/10.5802/aif. 2766.

FJR13 H. Fan, T. Jarvis, and Y. Ruan, The Witten equation, mirror symmetry, and quantum singularity theory, Ann. of Math. 178 (2013), no. 1, 1-106; https://doi.org/10.4007/annals. 2013.178.1.1.

Gat01 A. Gathmann, Gromov-Witten invariants of blow-ups, J. Algebraic Geom. 10 (2001), no. 3, 399-432.

Giv04 A.B. Givental, Symplectic geometry of Frobenius structures, Frobenius Manifolds, Aspects Math., vol. E36 (Friedr. Vieweg, Wiesbaden, 2004), 91-112; https://doi.org/10.1007/ 978-3-322-80236-1_4.

GP99 T. Graber and R. Pandharipande, Localization of virtual classes, Invent. Math. 135 (1999), no. 2, 487-518; https://doi.org/10.1007/s002220050293.

GW12 E. Gonzalez and C.T. Woodward, A wall crossing formula for Gromov-Witten invariants under variation of git quotient, 2012, arXiv:1208.1727.

GZK89 I. M. Gel'fand, A. V. Zelevinskii, and M. M. Kapranov, Hypergeometric functions and toric varieties, Funct. Anal. Appl. 23 (1989), no. 2, 94-106; https://doi.org/10.1007/BF01078777.

HH15 W. Q. He and J.X. Hu, Orbifold Gromov-Witten invariants of weighted blow-up at smooth points, Acta Math. Sin. (Engl. Ser.) 31 (2015), no. 5, 825-846; https://doi.org/10.1007/ s10114-015-3479-2.

Hu00 J. Hu, Gromov-Witten invariants of blow-ups along points and curves, Math. Z. 233 (2000), no. 4, 709-739; https://doi.org/10.1007/s002090050495.

ILLW12 Y. Iwao, Y.-P. Lee, H.-W. Lin, and C.-L. Wang, Invariance of Gromov-Witten theory under a simple flop, J. reine angew. Math. 663 (2012), 67-90; https://doi.org/10.1515/CRELLE. 2011.097.

Lee09 Y.-P. Lee, Notes on axiomatic Gromov-Witten theory and applications, Algebraic Geometry (Seattle 2005), Part 1, Proc. Sympos. Pure Math., vol. 80 (Amer. Math. Soc., Providence, RI, 2009), 309-323; https://doi.org/10.1090/pspum/080.1/2483940.

LLQW16 Y.-P. Lee, H.-W. Lin, F. Qu, and C.-L. Wang, Invariance of quantum rings under ordinary flops III: A quantum splitting principle, Camb. J. Math. 4 (2016), no. 3, 333-401; https: //doi.org/10.4310/CJM.2016.v4.n3.a2.

LPS16 Y.-P.-Lee, N. Priddis, and M. Shoemaker, A proof of the Landau-Ginzburg/Calabi-Yau correspondence via the crepant transformation conjecture, Ann. Sci. Éc. Norm. Supér. (4) 49 (2016), no. 6, 1403-1443; https://doi.org/10.24033/asens. 2312.

LR01 A.-M. Li and Y. Ruan, Symplectic surgery and Gromov-Witten invariants of Calabi-Yau 3folds, Invent. Math. 145 (2001), no. 1, 151-218; https://doi.org/10.1007/s002220100146.

Mil06 P. D. Miller, Applied asymptotic analysis, Grad. Stud. Math., vol. 75 (Amer. Math. Soc., Providence, RI, 2006); https://doi.org/10.1090/gsm/075.

MSH10 A. M. Mathai, R.K. Saxena, and H.J. Haubold, The H-function: theory and applications (Springer, New York, 2010); https://doi.org/10.1007/978-1-4419-0916-9.

PS16 N. Priddis and M. Shoemaker, A Landau-Ginzburg/Calabi-Yau correspondence for the mirror quintic, Ann. Inst. Fourier (Grenoble) 66 (2016), no. 3, 1045-1091; https://doi.org/10. 5802/aif. 3031.

Rei02 M. Reid, La correspondance de McKay, Astérisque (2002), no. 276, 53-72; http://www. numdam.org/item?id=SB_1999-2000__42__53_0. 


\section{QUANTUM COHOMOLOGY OF BLOWUPS}

Rua99 Y. Ruan, Surgery, quantum cohomology and birational geometry, Northern California Symplectic Geometry Seminar, Amer. Math. Soc. Transl. Ser. 2, vol. 196 (Amer. Math. Soc., Providence, RI, 1999), 183-198; https://doi.org/10.1090/trans2/196/09.

Tem13 N. M. Temme, Uniform asymptotic methods for integrals, Indag. Math. (N.S.) 24 (2013), no. 4, 739-765; https://doi.org/10.1016/j.indag.2013.08.001.

Tse10 H.-H. Tseng, Orbifold quantum Riemann-Roch, Lefschetz and Serre, Geom. Topol. 14 (2010), no. 1,1-81; https://doi.org/10.2140/gt.2010.14.1.

Zho08 J. Zhou, Crepant resolution conjecture in all genera for type A singularities, 2008, arXiv:0811.2023.

Pedro Acosta peacosta@umich.edu

Department of Mathematics, University of Michigan, Ann Arbor, MI 48109-1043, USA

Mark Shoemaker mark.shoemaker@colostate.edu

Department of Mathematics, Colorado State University, Fort Collins, CO 80523-1874 USA 